This item was submitted to Loughborough's Research Repository by the author.

Items in Figshare are protected by copyright, with all rights reserved, unless otherwise indicated.

\title{
Solitary waves with recirculation zones in axisymmetric rotating flows
}

PLEASE CITE THE PUBLISHED VERSION

PUBLISHER

(c) Cambridge University Press

LICENCE

CC BY-NC-ND 4.0

\section{REPOSITORY RECORD}

Derzho, Oleg G., and Roger H.J. Grimshaw. 2019. "Solitary Waves with Recirculation Zones in Axisymmetric Rotating Flows". figshare. https://hdl.handle.net/2134/2216. 


\title{
Solitary waves with recirculation zones in axisymmetric rotating flows
}

\author{
By OLEG DERZHO AND ROGER GRIMSHAW $\dagger$ \\ Department of Mathematics and Statistics, Monash University, Clayton, Vic 3800, Australia
}

(Received 11 November 1999 and in revised form 4 February 2002)

In this paper, we describe a theoretical asymptotic model for large-amplitude travelling solitary waves in an axially symmetric rotating flow of an inviscid incompressible fluid confined in an infinitely long circular tube. By considering the special, but important, case when the upstream flow is close to that of uniform axial flow and uniform rotation, we are able to construct analytical solutions which describe solitary waves with 'bubbles', that is, recirculation zones with reversed flow, located on the axis of the tube. Such waves have amplitudes which slightly exceed the critical amplitude, where there is incipient flow reversal. The effect of the recirculation zone is to introduce into the governing amplitude equation an extra nonlinear term, which is proportional to the square of the difference between the wave amplitude and the critical amplitude. We consider in detail a special, but representative, class of upstream inflow conditions. We find that although the structure of the recirculation zone is universal, the presence of such solitary waves is quite sensitive to the actual upstream axial and rotational velocity shear configurations. Our results are compared with previous theories and observations, and related to the well-known phenomenon of vortex breakdown.

\section{Introduction}

In this paper we are concerned with the structure of steady travelling solitary waves of large amplitude in the axially symmetric rotating flow of an inviscid, incompressible fluid contained in an infinitely long circular tube. There have been numerous studies of steady waves in such axisymmetric rotating flows, largely motivated by the possible relationship between such waves and the phenomenon of vortex breakdown. Most theoretical studies, and ours is no exception, are based on the Bragg-Hawthorne (Bragg \& Hawthorne 1950) equation, which is a single equation for the stream function incorporating the preservation of the flow circulation and head along streamlines. It was independently derived by Long (1953) (see also Squire 1956) and describes the distribution of the azimuthal component of vorticity. It is usually derived on the assumption that all streamlines originate upstream, so that there can be no 'bubble', that is, a recirculation zone with reversed flow, and that far upstream there is no wave component to the flow. For this situation, the Bragg-Hawthorne equation can be used to establish the existence of solitary waves of permanent form, which are maintained by a balance between nonlinearity and dispersion. However, the Bragg-Hawthorne equation may also allow solutions with recirculation zones, but additional assumptions are then required to determine the flow circulation and head there.

$\dagger$ Present address: Department of Mathematical Sciences, Loughborough University, Loughborough LE11 3TU. 
Originally the Bragg-Hawthorne equation was derived to relate inertial waves with the breakdown of the basic vortex flow. Thus, Squire (1956) suggested that inertial waves may propagate upstream, and cause a breakdown of the upstream vortex flow. Later, Benjamin $(1962,1967)$ proposed that vortex breakdown is a transition between two conjugate flow states, where it is assumed that the total circulation and head are preserved across the transition. With this assumption, Benjamin (1967) showed that in the absence of dissipation or transience, a force-free transition cannot be found. Instead, it was shown that the total head increases at the downstream state, and this extra head may generate unsteady waves there. Subsequently, Keller, Egli \& Exley (1985) and Keller (1995), amongst others, have shown that loss-free and force-free transitions are possible if the downstream conjugate flow includes an infinitely long 'bubble', or recirculation zone. This disturbance is stationary for a specific upstream swirl velocity determined by the upstream flow profile. The main approach used here was to obtain the downstream flow from conservation laws, and then to find numerically the transition zone connecting the upstream and downstream conjugate flow states. We will show inter alia in this paper that, in fact, this infinitely long recirculation zone is the limit of a class of solutions with recirculation zones of finite length.

In general, we conclude from various numerical simulations and observational studies that the recirculation zone found in axisymmetric vortex breakdown has finite size in both the radial and axial directions. However, in experiments, the observed finite size of the recirculation zone may be due to a combination of inertial effects and viscous diffusion at low Reynolds numbers, or to complicated instabilities inside and around the breakdown bubble at high Reynolds numbers. Theoretical models of this phenomenon have been proposed by Leibovich and collaborators (see, for instance, the review by Leibovich 1991). Thus, Leibovich \& Kribus (1990) established the existence of four types of solution: multiple columnar solutions corresponding to the conjugate states of Benjamin $(1962,1967)$, solitary waves, periodic wavetrains and solitary waves superimposed on the conjugate flow that emerges from the wavetrain. They also claimed that weakly nonlinear solitary wave theory can be accurate even in the case of quite large-amplitude waves, containing regions of flow reversal. The crucial, and explicit, assumption of Leibovich \& Kribus (1990) is that the functional form of the Bragg-Hawthorne equation is identical inside and outside the recirculation zone. As we discuss later in this paper, this assumption leads, in general, to unstable solutions in the recirculation zone, even in the axisymmetric context. Further, we will show that the assumption that the same functional form holds throughout the flow regime, including any recirculation zones, leads to a prediction of vortex breakdown in a supercritical flow for the case of a uniformly rotating fluid with uniform axial velocity, contained in an infinitely long straight cylinder. Such a prediction is contrary to the theoretical results of Fraenkel (1964) and Keller et al. (1985), and has not been found in numerical simulations (see, for instance, Hanazaki 1996).

In this study, we construct solitary waves with recirculation zones, in which we make the alternative assumption that the flow in the recirculation zone is stagnant, at least to leading order with respect to the small parameter which defines the size of the recirculation zone. Indeed, this is required by the Prandtl-Batchelor (Batchelor 1956) condition for steady axially symmetric flow in the limit of vanishing viscosity. Our assumption of a near-stagnant flow inside the recirculation zone is consistent with the earlier studies by Keller et al. (1985) and Wang \& Rusak (1997a), where the same approach was used. Our asymptotic solution technique is valid for the important special case when the upstream flow has nearly uniform axial velocity, and nearly 
uniform angular velocity. The crucial circumstance here is that in the limit when the upstream flow is exactly uniform, the Bragg-Hawthorne equation is a linear equation even for waves of finite amplitude. This result has been exploited by Grimshaw \& Yi (1993) who constructed an integro-differential equation to describe the slow evolution of finite-amplitude waves up to the onset of incipient flow reversal. In this paper, we consider steady waves, whose amplitudes exceed the critical amplitude where flow reversal first occurs and which contain recirculation zones. Our asymptotic technique is based on that used by Derzho \& Grimshaw (1997) for the closely analogous problem of large-amplitude waves in a stratified fluid (see also, Rottman, Broutman \& Grimshaw (1996) and Aigner, Broutman \& Grimshaw (1999) for related numerical simulations). Our main results are the construction of an asymptotic model for solitary waves with recirculation zones, for a wide class of inflow conditions which are close to the state of uniform axial flow and uniform angular velocity. The structure of the recirculation zone is described in detail. The present asymptotic model is related to other theories, and its implications for the phenomena of vortex breakdown are discussed.

We must point out that our model is formulated to describe steady travelling solitary wave disturbances in a tube of infinite length. Such a formulation does not exactly correspond to the experimental set-ups described in Sarpkaya (1971), Leibovich (1984) and Escudier (1988), or to the numerical simulations by Beran \& Culick (1992), Lopez (1994) and Hanazaki (1996). In all of these numerical studies, breakdown of the approaching vortex was induced by a local contraction of the tube, so that the separation zone was observed in the lee of that contraction. Also, a local contraction was used by Beran \& Culick (1992) to prevent the formation of a recirculation eddy at the upstream boundary, which has often been noted since the first numerical study of axisymmetric breakdown by Kopecky \& Torrance (1973). However, a local contraction is not the only way to initiate vortex breakdown; Brown \& Lopez (1990) observed a downstream widening of the vortex owing to viscous dissipation that eventually induces vortex breakdown. Further, Darmofal (1996) and Snyder \& Spall (2000) claimed a reasonably good agreement with the relevant experiments of Faler \& Leibovich (1978) and Sarpkaya (1971). They did not use a local contraction to induce the breakdown, but carefully modelled both the flow field in the approaching vortex (Snyder \& Spall started with the vortex shedding from the vane generator). Snyder \& Spall (2000) also performed calculations with inlet conditions of zero radial velocity, and fixed swirl and axial velocity profiles that were obtained from the solution of the problem with the exact modelling of the flow past a set of turning vanes. No significant difference was observed between these two calculations. This result may support the idea that there are no upstream advancing disturbances in their calculations at least far upstream of the breakdown bubble, and the conditions for the velocity field are fairly accurate. We point out that in all these successful calculations the divergence of the tube (at least in the region where a separation bubble was observed experimentally) was carefully modelled. In the case of a confined geometry, where the boundary conditions are obvious, Lopez (1990) has shown excellent agreement with the relevant experiments.

Thus, we infer that, to predict experiments successfully, we need to know the exact inflow, outflow and tube boundary conditions. In part, these conditions are determined by the flow field in the approaching vortex. The rest of the conditions depend on how the approaching vortex is disturbed while propagating through the tube, whose cross-section may vary in the axial direction. In order to understand how to manage vortex breakdown, we can split the problem into three parts. First, it is important to 
identify the relevant flow profiles which enlarge or reduce the size of the separation bubble, or which may allow multiple flow states, etc. The second set of problems is how to create these profiles, or how to disturb the approaching vortex to reach the desired result. In this paper, we do not deal with the second set of problems. The third part is to identify those profiles which may lead to a stationary and stable vortex. We have concentrated on the ideal boundary conditions of an infinite straight tube, where a disturbance to the given approaching vortex is assumed to vanish upstream, and examined what kind of disturbance of permanent form may propagate on the given approaching flow, that is, which approaching flows allow disturbances with a separation zone, multiple states, or for which cases stationary solitary wave solutions do not exist.

We must point out that our formulation is different from the studies of Buntine \& Saffman (1995) (steady problem), and the studies by Wang \& Rusak (1997a), Rusak, Wang \& Whiting (1998) (unsteady problem) where the major effects are due to the constricted geometry of the tube. The inlet boundary conditions in Wang \& Rusak (1997a,b), Rusak et al. (1998) do not fix the radial velocity, but instead fix the azimuthal vorticity. To this end they allow the radial velocity to be freely adjusted to reflect the upstream influence which is allowed to reach the inlet in their model. The model of Rusak et al. (1998), although for a straight tube, was nevertheless claimed to be relevant to the studies of Beran \& Culick (1992), Lopez (1994) and Darmofal (1996), where the condition of zero radial velocity at the inlet was used. However, the inlet conditions of Rusak et al. (1998) do not contain the length scale of a local contraction (Beran \& Culick 1992; Lopez 1994), or the length scale associated with a divergence of the tube (Darmofal 1996). These effects due to the tube profile are important in the problem studied by these authors, not only because these tube profiles prevent the changes of the inlet conditions (which the conditions of Rusak et al. (1998) also do), but also because the properties of the generated wave are affected by the exact shape of the tube. Also, it can be seen that to obtain successful unsteady numerical simulations of the experiments by Faler \& Leibovich (1978), the outlet conditions while being similar to those of Rusak et al. (1998), (i.e. setting negligible axial gradients), were preceded by a convergent section of the tube, thus re-accelerating the flow near the exit (Darmofal 1996). In simulations attempted without this section, an iterative process of finding the solution was found to be unstable (Darmofal 1996). Note, however, that Lopez (1994) did not need to use a convergent section near the end. All these results probably indicate that the outlet conditions should be chosen with care according to each particular case.

Another recent set of successful numerical calculations (Snyder \& Spall 2000) reveal that the resulting flow was supercritical near the outlet boundary, thus minimizing the upstream propagation of axisymmetric waves generated at the outlet. Hanazaki (1996), also in a numerical study, used a transparent boundary condition at the outlet, which together with the sufficiently long numerical domain minimizes the influence of the outlet condition on the breakdown. In the numerical simulations of Rusak et al. (1998), a separation bubble occurs at the outlet boundary. Wang \& Rusak (1997a) reported that their inviscid steady solutions are supercritical at the outlet, so that their condition of 'no axial gradient' may be used in that case. However, such a condition may cause artificial reflections if disturbances can propagate in both directions. This could be the case for the temporal problem, in the interior of the recirculation 'bubble' where the motion is generally very complicated.

Despite these highlighted differences, the studies of Rusak et al. (1998) and Wang 
\& Rusak (1997a) unite most of the recent numerical studies, and give an explanation of the existence of two critical swirl values, as observed by Beran \& Culick (1992), Beran (1994) and Lopez (1994). The lower value corresponds to the special solution of Keller et al. (1985), and the higher value corresponds to the critical swirl originally identified by Benjamin (1962). Rusak et al. (1998) linked these critical values of swirl with the stability characteristics of the flow in a constricted tube, and they describe the vortex breakdown in high-Reynolds-number flows as a process of the evolution of the initial columnar vortex to another relatively stable and lower-energy equilibrium state around a large stagnant separation zone. Wang \& Rusak (1996) describe such evolution as a result of the interaction between upstream advancing disturbances and the approaching flow, which leads to a loss of stability of the base columnar state when the swirl is near or above the critical level.

Again, we remind the reader that our study is different from most of the studies mentioned above, and is not directly relevant to the existing experimental set-ups, as we are describing disturbances in an infinite tube, where there is a simple and natural way to impose upstream boundary conditions. Our paper describes a novel way of constructing an asymptotic solution for an inertial solitary wave with a recirculation zone. It is also pertinent to note that this approach is capable of allowing several different sets of upstream boundary axial and swirl velocity profiles. In applying our results to the vortex breakdown phenomenon, our main purpose is to identify, albeit in this ideal formulation, which profiles in the approaching vortex may enhance breakdown and which may suppress it. Our theoretical model is capable of predicting multiple-state solutions, the flow around the separation bubble, as well as its shape, from a relatively simple ordinary differential equation, rather than from a complicated numerical model. Finally, we point out that a systematic stability analysis of these travelling solitary wave solutions is beyond the scope of the present study.

In $\S 2$, we formulate the steady equations of motion and identify all relevant small parameters. In $\S 3$, we describe the asymptotic construction of large-amplitude solitary waves with recirculation zones. In $\S 4$, we discuss our solutions in more detail, and for several specific cases of upstream flow conditions. Then, in $\S 5$, we present a concluding discussion, and relate our results to the phenomenon of vortex breakdown.

\section{Formulation}

We consider the axisymmetric, steady flow of an inviscid, non-diffusive swirling fluid of constant density, confined to a tube of circular cross-section. In standard notation, in a frame of reference moving with a wave of speed $c$, the equations of motion are,

$$
\begin{gathered}
u_{x}+\frac{1}{r}(r v)_{r}=0, \\
(u-c) u_{x}+v u_{r}+p_{x}=0, \\
(u-c) v_{x}+v v_{r}-\frac{w^{2}}{r}+p_{r}=0, \\
(u-c) w_{x}+v w_{r}+\frac{v w}{r}=0 .
\end{gathered}
$$

Here, the coordinates are $(x, r, \theta)$ with $x$ directed along the tube axis, $(u, v, w)$ are the corresponding velocity components, and $p$ is the pressure (see figure 1). Next, we 


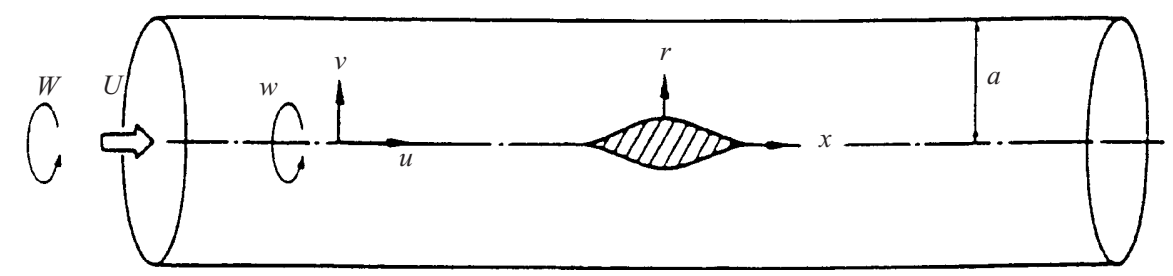

FIGURE 1. Coordinate scheme.

introduce the streamfunction $\psi$ in the usual way,

$$
u-c=\frac{\psi_{r}}{r}, \quad v=-\frac{\psi_{x}}{r} .
$$

Equation (2.1d) shows that the circulation is constant along a streamline, so that

$$
r w=C(\psi),
$$

where the functional form of $C(\psi)$ is to be determined from the inflow distribution of circulation for those streamlines which originate upstream. Inside the recirculation zone, which is located on the tube axis, we will show later that the circulation is very weak, and, in effect, $C(\psi)=0$ there. Finally, it can be shown that the two momentum equations $(2.1 b)$ and $(2.1 c)$ yield a single equation for the stream function,

$$
\psi_{x x}+\psi_{r r}-\frac{\psi_{r}}{r}+C(\psi) C^{\prime}(\psi)=r^{2} G(\psi),
$$

where $G(\psi)$ is the derivative of the total head function, and is also to be determined from the inflow conditions for those streamlines which originate upstream. Inside the recirculation zone, we shall argue later that the flow is nearly stagnant, and so $G(\psi) \approx 0$ there. Equation (2.4) is the Bragg-Hawthorne equation for the azimuthal vorticity used in numerous studies of rotating flows. The circular tube has radius $a$, and the boundary condition at $r=a$ is $\psi=$ constant.

It is convenient to introduce dimensionless variables based on a typical axial velocity $U_{0}$ and the radius

$$
\psi^{\prime}=\frac{2 \psi}{U_{0} a^{2}}, \quad r^{\prime}=\frac{r}{a}, \quad x^{\prime}=\frac{x}{a} .
$$

Hereinafter, we will omit the primes. Further, we will assume that the inflow axial velocity is nearly uniform, and the inflow rotation is likewise nearly uniform. Thus, we suppose that the inflow conditions are, in dimensionless form,

$$
\begin{gathered}
u \rightarrow u_{\infty}=1+\kappa U(\xi) \quad \text { as } \quad x \rightarrow-\infty, \\
\frac{w}{r} \rightarrow \frac{w_{\infty}}{r}=\Omega_{0}(1+\sigma \Omega(\xi)) \quad \text { as } \quad x \rightarrow-\infty,
\end{gathered}
$$

where,

$$
\xi=r^{2} .
$$

Here, $\kappa, \sigma$ are small parameters, characterizing the small departures from uniform axial flow and uniform rotation, respectively. It follows that

$$
\psi=\xi(1-c)+\kappa \int_{0}^{\xi} U(\hat{\xi}) \mathrm{d} \hat{\xi} \quad \text { as } \quad x \rightarrow-\infty .
$$

For those streamlines which originate upstream, we can now determine the functions 
$C(\psi)$ and $G(\psi)$. We find that

$$
\begin{gathered}
C(\psi)=\frac{\Omega_{0}}{1-c}\left\{\psi+\sigma \psi \Omega\left(\frac{\psi}{1-c}\right)-\kappa \int^{\psi / 1-c} U(\hat{\xi}) \mathrm{d} \hat{\xi}+\cdots\right\}, \\
G(\psi)=\frac{2 \Omega_{0}^{2}}{1-c}\left\{1-\frac{\kappa U(\psi / 1-c)}{1-c}+2 \sigma \Omega\left(\frac{\psi}{1-c}\right)+\frac{\sigma \psi}{1-c} \Omega^{\prime}\left(\frac{\psi}{1-c}\right)\right\} \\
+2 \kappa U^{\prime}\left(\frac{\psi}{1-c}\right)+\cdots .
\end{gathered}
$$

Here, the omitted terms are relatively of $o(\kappa, \sigma)$. Using the expressions $(2.8 a)$ and $(2.8 b)$, equation (2.4) can be rewritten in explicit form in terms of the perturbed streamfunction $\phi$ where

$$
\psi=\xi(1-c)+\kappa \int_{0}^{\xi} U(\hat{\xi}) \mathrm{d} \hat{\xi}+\phi .
$$

After some algebra, we find that

where

$$
\phi_{x x}+4 \xi \phi_{\xi \xi}+\lambda^{2} \phi=F(\phi),
$$

$$
\lambda=\frac{2 \Omega_{0}}{|1-c|},
$$

and

$$
\begin{aligned}
F(\phi)= & \kappa \lambda^{2}\left\{\int_{\xi}^{\xi+\phi / 1-c} U(\hat{\xi}) \mathrm{d} \hat{\xi}+\frac{\phi}{1-c} U\left(\xi+\frac{\phi}{1-c}\right)\right\}-\sigma \lambda^{2}\left\{2 \phi \Omega\left(\xi+\frac{\phi}{1-c}\right)\right. \\
& \left.+\phi\left(\xi+\frac{\phi}{1-c}\right) \Omega^{\prime}\left(\xi+\frac{\phi}{1-c}\right)\right\}+4 \kappa \xi\left\{U^{\prime}\left(\xi+\frac{\phi}{1-c}\right)-U^{\prime}(\xi)\right\}+\cdots .
\end{aligned}
$$

Here, $\Omega_{0}$ is the swirl number, which is proportional to the ratio of the azimuthal velocity at the radius of the bounding tube wall, to the axial velocity along the centreline. The boundary conditions on the tube axis and wall are that

$$
\phi=0 \quad \text { at } \xi=0,1 .
$$

The boundary condition at $\xi=0$ derives from the requirement that the flow be finite and regular there. The boundary condition at the tube wall $\xi=1$ is to ensure that there is no flow through the tube wall, and this is the only boundary condition that can be imposed there in our present inviscid formulation. Note that we are at liberty, if need be, to suppose that the tube wall is corotating with the fluid and has angular velocity close to $\Omega$, thus enabling us to make a comparison with experiments such as those of Pritchard (1969). Further, our inviscid formulation can be effectively compared with the numerical simulations, such as those of Hanazaki (1996), who use free-slip conditions at the tube wall. Next, in the upstream and downstream directions we require that

$$
\phi \rightarrow 0 \quad \text { as } \quad x \rightarrow \pm \infty .
$$

In the absence of any recirculating zone, (2.10), (2.11) and (2.12) provide a well-posed formulation of the problem, since then it is assumed that all streamlines originate upstream. Next, we note that if

$$
1+\frac{\phi_{\xi}}{1-c}>0
$$


everywhere, then, to leading order with respect to the small parameter $\kappa, u-c>0(<0)$ according as $c<1(>1)$ and there is no flow reversal at any point in the flow.

Here, we are concerned with the case when the condition (2.13) is violated on the tube axis. Then, the critical condition

$$
1-c+\phi_{\xi}=0 \quad \text { at } \quad \xi=0,
$$

defines a critical wave amplitude, and we shall show that for waves with amplitudes greater than this critical amplitude, there is a zone of recirculating fluid located on the tube axis. We shall suppose that the boundary of this vortex core is given by (see figure 2)

$$
r=\eta(x),
$$

where $\eta(x)=0$ for $|x|>x_{0}$. Outside this recirculation zone, the flow is determined by (2.10), with the boundary conditions

$$
\phi+\eta^{2}(1-c)+\kappa \int_{0}^{\eta^{2}} U(\hat{\xi}) \mathrm{d} \hat{\xi}=0 \text { at } \xi=\eta^{2},
$$

and

$$
\phi=0 \quad \text { at } \xi=1,
$$

as well as the conditions (2.12) at infinity. Inside the recirculation zone, the governing equation is again (2.4), but now the circulation function $C(\psi)$ and the total head derivative $G(\psi)$ are yet to be determined. However, we shall show that in the recirculation zone both $C(\psi)$ and $G(\psi)$ are approximately zero. That is, the flow there is approximately stagnant and without rotation. This is consistent with the observations of Faler \& Leibovich (1978), and Escudier (1988), and is similar to the hypothesis used by Wang \& Rusak (1997a). Note that simple analytic continuation of $C(\psi)$ and $G(\psi)$ across the boundary of the recirculation zone does not generally lead to physically meaningful solutions, and hence we can anticipate that both $C(\psi)$ and $G(\psi)$ must be determined by other, physically relevant, considerations (cf. Keller 1995). Finally, since the boundary of the recirculation zone, $r=\eta(x)$, is to be determined as part of the full solution, it is necessary to impose the dynamic condition that the pressure is continuous across the recirculation zone boundary, as well as the kinematic condition $(2.16 a)$. It can be shown that pressure continuity is equivalent to the continuity of $\psi_{\xi}$,

$$
\left[\psi_{\xi}\right]=0 \text { at } \xi=\eta^{2} .
$$

Henceforth, we shall refer to the region where $r>\eta>0$ as the inner zone, i.e. $|x|<x_{0}$. The region where $\eta \equiv 0$ is then the outer zone, i.e. $|x|>x_{0}$. Note that the case when $x_{0}=0$ is the particular case when there is no inner zone, and the outer zone occupies the whole domain. This particular case corresponds to the existence of inertial waves without any flow reversals.

\section{Derivation of the amplitude equation}

\subsection{Solution in the outer zone}

We shall seek solitary wave solutions whose width is much greater than the radius of the tube. Hence, it is convenient to introduce the small parameter $\varepsilon$ and let

$$
X=\varepsilon x
$$

be the new axial variable. Later, it will emerge that the small parameters $\kappa$ and $\sigma$ are both $O\left(\varepsilon^{2}\right)$ to achieve an optimal balance, and so we put $\kappa=\hat{\kappa} \varepsilon^{2}, \sigma=\hat{\sigma} \varepsilon^{2}$. Then, we 


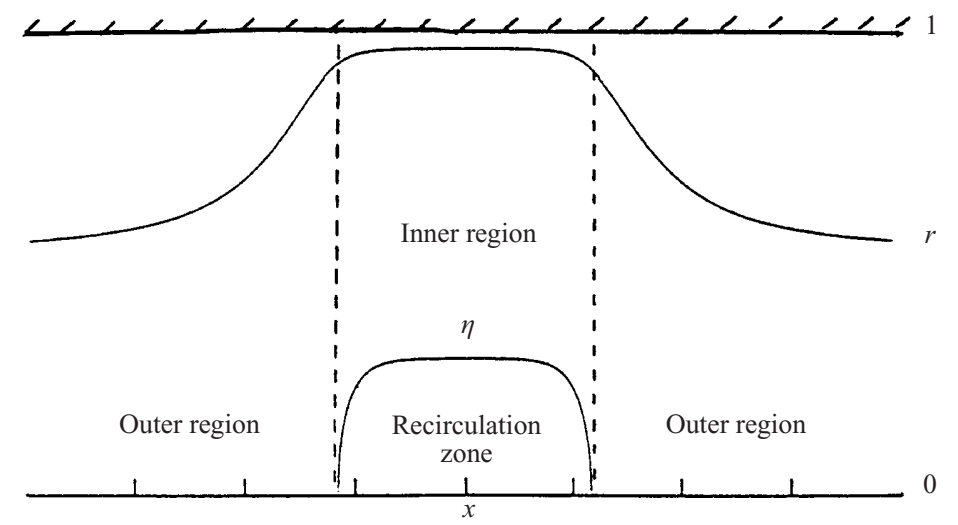

FIGURE 2. Sketch of the outer, inner and recirculation zones.

seek a solution in terms of the asymptotic expansions

$$
\begin{gathered}
\phi=\phi^{(0)}(X, \xi)+\varepsilon^{2} \phi^{(1)}(X, \xi)+\cdots, \\
c=c^{(0)}+\varepsilon^{2} c^{(1)}+\cdots .
\end{gathered}
$$

These expansions are substituted into (2.10a), and the boundary conditions (2.11). At the lowest order we find that

$$
\phi^{(0)}=A(X) W(\xi)
$$

where

$$
W(\xi)=r J_{1}\left(\lambda^{(0)} r\right),
$$

and

$$
J_{1}\left(\lambda^{(0)}\right)=0 .
$$

Here, $J_{1}(\langle)$ is the Bessel function of order 1 , and

$$
\lambda^{(0)}=\left|\frac{2 \Omega_{0}}{1-c^{(0)}}\right| .
$$

For the lowest mode, $\lambda^{(0)}=3.83$, and we shall confine our attention to this mode henceforth. The higher modes may be considered in a similar way, but, by analogy with the corresponding stratified flow problem (Akylas \& Grimshaw 1992), we expect that these higher modes do not exist in a purely local form owing to the generation of lower-mode radiation.

The amplitude $A(X)$ is now determined at the next order in the expansion, and we find that

where

$$
4 \xi \phi_{\xi \xi}^{(1)}+\lambda^{(0)^{2}} \phi^{(1)}=F^{(1)},
$$

$$
F^{(1)}=-A_{X X} W+\frac{F\left(\phi^{(0)}\right)}{\varepsilon^{2}}+\frac{2 \lambda^{(0)^{2}} c^{(1)}}{\left(c^{(0)}-1\right)} A W .
$$

Here, $F\left(\phi^{(0)}\right)$ is determined from $(2.10 c)$ and is $O\left(\varepsilon^{2}\right)$, since $\kappa$ and $\sigma$ are $O\left(\varepsilon^{2}\right)$. This is to be solved with the boundary conditions

$$
\phi^{(1)}=0 \quad \text { at } \quad \xi=0,1 .
$$


A compatibility condition is required, and is here given by

$$
\int_{0}^{1} F^{(1)} W \frac{\mathrm{d} \xi}{2 \xi}=0 .
$$

Substitution of (3.5b) into (3.7) finally yields the amplitude equation

$$
-A_{X X}+\Delta A+M(A)=0,
$$

where

$$
\begin{gathered}
\Delta=\frac{2 \lambda^{(0)^{2}} c^{(1)}}{\left(c^{(0)}-1\right)} \\
M(A)=\hat{\kappa} M_{\kappa}(A)+\hat{\sigma} M_{\sigma}(A), \\
I M_{\kappa}(A)=\lambda^{(0)^{2}} \int_{0}^{1} \frac{W \mathrm{~d} \xi}{2 \xi}\left\{\int_{\xi}^{\xi+\phi^{(0)} / 1-c^{(0)}} U(\hat{\xi}) \mathrm{d} \hat{\xi}+\frac{\phi^{(0)}}{\left(1-c^{(0)}\right)} U\left(\xi+\frac{\phi^{(0)}}{1-c^{(0)}}\right)\right\} \\
+\int_{0}^{1} 2 W \mathrm{~d} \xi\left\{U^{\prime}\left(\xi+\frac{\phi^{(0)}}{1-c^{(0)}}\right)-U^{\prime}(\xi)\right\}, \\
I M_{\sigma}(A)=-\lambda^{(0)^{2}} \int_{0}^{1} \frac{W \mathrm{~d} \xi}{2 \xi}\left\{2 \phi^{(0)} \Omega\left(\xi+\frac{\phi^{(0)}}{1-c^{(0)}}\right)\right. \\
\left.+\phi^{(0)}\left(\xi+\frac{\phi^{(0)}}{1-c^{(0)}}\right) \Omega^{\prime}\left(\xi+\frac{\phi^{(0)}}{1-c^{(0)}}\right)\right\},
\end{gathered}
$$

and

$$
I=\int_{0}^{1} \frac{W^{2} \mathrm{~d} \xi}{2 \xi}=\int_{0}^{1} r J_{1}^{2}\left(\lambda^{(0)} r\right) \mathrm{d} r=\frac{1}{2} J_{0}\left(\lambda^{(0)}\right)^{2} .
$$

The nonlinear term $M(A)$ depends on the amplitude $A$ in a complicated manner dependent on the form of the functions $U(\xi)$ and $\Omega(\xi)$, where we recall that these represent the small departures from uniform axial flow and uniform rotation, respectively. We shall give some simple illustrative examples of the calculation of $M(A)$ below. The amplitude equation (3.8a) is a steady-state generalized Korteweg-de Vries $(\mathrm{KdV})$ equation, and is the steady-state version of the finite-amplitude long-wave evolution equation derived by Grimshaw \& Yi (1993).

The condition (2.13) which excludes flow reversals at any point in the flow is now satisfied, to leading order in $\varepsilon$, provided that

$$
\frac{2}{\lambda^{(0)}\left|J_{0}\left(\lambda^{(0)}\right)\right|}>\frac{A}{1-c^{(0)}}>-\frac{2}{\lambda^{(0)}} .
$$

Here, the upper limit corresponds to flow reversal at the tube wall $(\xi=1)$, whereas the lower limit corresponds to flow reversal on the tube axis $(\xi=0)$. We are concerned here with this latter case, and so the critical amplitude $A_{*}$ for wave breakdown and flow reversal is given by

$$
A_{*}=-\frac{2\left(1-c^{(0)}\right)}{\lambda^{(0)}}= \pm \frac{4\left|\Omega_{0}\right|}{\lambda^{(0)^{2}}} .
$$

Here, the alternative signs are those corresponding to $c^{(0)}>1(<1)$, and so consequently $A_{*}>0(<0)$. Since $W(\xi)>0$ for $0<\xi<1$, it follows that wave breakdown on the tube axis occurs for waves whose axial flux is positive (negative) when $c^{(0)}>1(<1)$. We also note here, for the present case of a straight tube, that since 
$\left|J_{0}\left(\lambda^{(0)}\right)\right| \approx 0.4(<1)$, the critical amplitude for wave breakdown on the axis is always less in absolute value than that for wave breakdown on the tube wall, although the latter has the opposite sign. It is consistent with the results of numerical simulations by Beran \& Culick (1992), Lopez (1994), Hanazaki (1996) and Snyder \& Spall (2000) among others.

To conclude this subsection, we calculate the form of the nonlinear terms $M(A)$ when the perturbation inflow axial velocity $U(\xi)$, and angular velocity $\Omega(\xi)$ are quadratic polynomials in $\xi$, given by

$$
\begin{aligned}
& U=a_{1}(1-\xi)+a_{2}\left(1-\xi^{2}\right), \\
& \Omega=b_{1}(1-\xi)+b_{2}\left(1-\xi^{2}\right) .
\end{aligned}
$$

Here, the coefficients are chosen to enforce $U(1)=\Omega(1)=0$. It is straightforward to show that then

$$
M(A)=\alpha_{1} A+\alpha_{2} A^{2}+\alpha_{3} A^{3},
$$

where $\alpha_{1}, \alpha_{2}$ and $\alpha_{3}$ are each represented by certain linear (although complicated) expressions in the coefficients $a_{1}, a_{2}, b_{1}$ and $b_{2}$ (see Appendix). Note that the coefficient $\alpha_{1}$ can be absorbed into the detuning parameter $\Delta$. When the nonlinear term is given by (3.12), the amplitude equation (3.8a) reduces to the steady-state version of the modified $\mathrm{KdV}$ equation, whose periodic and solitary-wave solutions are well-known. For instance, the solitary-wave solution is

$$
A=\frac{A_{0}}{1+B_{0} \cosh \left\{\gamma\left(X-X_{*}\right)\right\}},
$$

where

$$
A_{0}=-\frac{3 \gamma^{2}}{\alpha_{2}}, \quad B_{0}^{2}=1-\frac{9 \alpha_{3} \gamma^{2}}{2 \alpha_{2}^{2}}, \quad \Delta+\alpha_{1}=\gamma^{2},
$$

where $X_{*}$ is an arbitrary phase, to be determined later from matching conditions with the inner zone. The wave amplitude (peak value) is $a_{0}=A_{0} /\left(1+B_{0}\right)$, where the polarity of the wave is defined by the sign of $-\alpha_{2}\left(1+B_{0}\right)$ (since $\left.\alpha_{2} A_{0}<0\right)$. When $\alpha_{3}>0$, then $0<B_{0}<1$ and for small wave amplitudes $\left(B_{0} \rightarrow 1\right)$, the solitary wave (3.13a) resembles the familiar KdV solitary wave

$$
A=a_{0} \operatorname{sech}^{2}\left(\sqrt{-\frac{1}{6} \alpha_{2} a_{0}}\left(X-X_{*}\right)\right) .
$$

On the other hand, as $B_{0} \rightarrow 0$, the wave amplitude approaches a limiting critical value $-2 \alpha_{2} / 3 \alpha_{3}$, while the width increases to infinity, and the wave becomes the so-called 'thick' solitary wave. When $\alpha_{3}<0$, there are two families of solitary waves, both with $B_{0}^{2}>1$. The first has $1<B_{0}<\infty$, and has the opposite polarity to the KdV solitary wave (3.14), whereas for large amplitudes $\left(B_{0} \rightarrow \infty\right)$ it approaches the solitary-wave solution of the cubic $\mathrm{KdV}$ equation (i.e. $\alpha_{2}=0$ ) given here by

$$
A=a_{0} \operatorname{sech}\left(\sqrt{-\frac{1}{2} \alpha_{3} a_{0}^{2}}\left(X-X_{*}\right)\right) .
$$

The other family has $-\infty<B_{0}<-1$; as $B_{0} \rightarrow-\infty$ it again resembles (3.15) but with opposite polarity, but now as $B_{0} \rightarrow-1$ the amplitude decreases to another critical value, $-4 \alpha_{2} / 3 \alpha_{3}$, and in this limit becomes an algebraic solitary wave,

$$
A=\frac{-12 \alpha_{2}}{9 \alpha_{3}-2 \alpha_{2}^{2}\left(X-X_{*}\right)^{2}}, \quad \Delta+\alpha_{1}=0 .
$$


These results for this special case when $M(A)$ is given by (3.12) can now be combined with the criterion (3.9) for the exclusion of any flow reversals. First, suppose that $\alpha_{3}>0$, and consider the case when $A_{*}>0(<0)$ (i.e. $c^{(0)}>1(<1)$ ). Then, solitary waves without flow reversals exist whenever $-A_{*} /\left|J_{0}\left(\lambda^{(0)}\right)\right|<a_{0}<A_{*}$, $\left(-A_{*} /\left|J_{0}\left(\lambda^{(0)}\right)\right|>a_{0}>A_{*}\right)$, where we note that $\left|J_{0}\left(\lambda^{(0)}\right)\right| \approx 0.4$. Thus, since $\alpha_{2} a_{0}<0$, it follows that flow reversal will occur on the axis whenever $\alpha_{2} A_{*}<0$, and also $2\left|\alpha_{2}\right|>3 \alpha_{3}\left|A_{*}\right|$. Next, let $\alpha_{3}<0$, and again consider the case when $A_{*}>0(<0)$. Now, the criterion for solitary waves without flow reversals to exist is the same as that stated above for the case $\alpha_{3}>0$, but the allowed ranges for the wave amplitude $a_{0}$ differ. For the first family, which has opposite polarity to $\alpha_{2}$, it follows again that flow reversal will occur on the axis whenever $\alpha_{2} A_{*}<0$. For the second family, which has the same polarity as $\alpha_{2}$, flow reversal can occur on the axis whenever $\alpha_{2} A_{*}>0$ and also $4\left|\alpha_{2}\right|<3\left|\alpha_{3} A_{*}\right|$. The case $\alpha_{2}=0$ is obtained in an obvious way as a limiting case for each family.

When $M(A)$ contains nonlinear terms of higher-order than the cubic term in (3.12), analogous solutions can be constructed, and have been discussed by Derzho (1992) and Clarke \& Grimshaw (1999).

\subsection{Solution in the inner zone}

This is the region $|x|<x_{0}$, where $r>\eta(x)>0$ (see (2.15) and figure 1). The recirculation zone itself is the region where $0 \leqslant r<\eta(x)$ and $|x|<x_{0}$. We shall suppose that the recirculation zone has a long length scale and a small radius, so that we may put

where

$$
\eta=\delta f(z)
$$

$$
z=\beta x .
$$

Here, $\beta$ and $\delta$ are both small parameters whose relationship to the governing small parameter $\varepsilon$ will be determined below. The boundaries of the recirculation zone $|x|=x_{0}$ can now be written as $|X|=X_{0}$, or $|z|=z_{0}$, where $X_{0}=\varepsilon x_{0}, z_{0}=\beta x_{0}$ and so $X_{0}=\varepsilon z_{0} / \beta$. The width of the inner zone, $2 z_{0}$, is a parameter to be determined as part of our asymptotic construction. It turns out that $z_{0}$ is of order unity, and so $\varepsilon \ll \beta$. Thus, the inner zone is wide compared to the tube radius, but is smaller than the overall length scale of the wave.

Outside the recirculation zone, we again seek a solution of the form (3.2), but with the slow variable $X$ replaced by $z(3.17 b)$,

$$
\phi=\phi^{(0)}(z, \xi)+\varepsilon^{2} \phi^{(1)}(z, \xi)+\cdots,
$$

where

$$
\phi^{(0)}=A(z) W(\xi) .
$$

Further, we shall assume that in this inner zone the amplitude $A(z)$ is close to the critical amplitude $A_{*}(3.10)$, so that

$$
A(z)=A_{*}+\mu B(z) \operatorname{sign} A_{*},
$$

where $\mu$ is another small parameter whose relationship to the governing small parameter $\varepsilon$ will be determined below. Note that $A_{*}>0(<0)$ according as $c^{(0)}>0(<0)$, and correspondingly we can assume that $B(z)>0$ with a maximum value for $B(z)$ of 1 , so that the maximum wave amplitude is $\left|A_{*}\right|+\mu$. Further, it is pertinent to note 
here that since we shall require $\phi_{x x}^{(0)}$ to be $O\left(\varepsilon^{2}\right)$, it follows that

$$
\mu \beta^{2}=\varepsilon^{2} \text {. }
$$

Then, on substituting (3.18) into (2.10a), and using (3.19) we obtain at the next order

$$
4 \xi \phi_{\xi \xi}^{(1)}+\lambda^{(0) 2} \phi^{(1)}=G^{(1)},
$$

where

$$
G^{(1)}=\mp B_{z z} W+\Delta A_{*} W+\frac{F\left(A_{*} W\right)}{\varepsilon^{2}},
$$

and

$$
\phi^{(1)}=0 \quad \text { at } \quad \xi=1 .
$$

Hereinafter the alternative signs refer to $A_{*}>0(<0)$. The boundary condition at $\xi=\delta^{2} f^{2}(z)$ is determined by matching with the solution in the recirculation zone, using the conditions $(2.16 a)$ and (2.17). This process will be described in the next paragraph, but we note here that because $\delta \ll 1$, the boundary at $\xi=\delta^{2} f^{2}(z)$ may be asymptotically relocated to $\xi=0$. Then, a compatibility condition similar to (3.7) is imposed on $(3.21 a)$ with the result that

$$
\mp B_{z z}+\Delta A_{*}+M\left(A_{*}\right)=\frac{\phi^{(1)}(z, 0) \lambda^{(0)}}{I} .
$$

We shall now turn to the boundary conditions (2.16a) and (2.17). First, consider the kinematic boundary condition (2.16a). Using (3.17a), (3.18) and (3.19) we find that, at the leading order

$$
\varepsilon^{2} \phi^{(1)}(z, 0) \pm \frac{1}{2} \mu \delta^{2} \lambda^{(0)} f^{2} B-\frac{1}{16} \delta^{4} A_{*} \lambda^{(0)^{3}} f^{4}=0 .
$$

Clearly, this expression now requires the following balance of parameters

$$
\varepsilon^{2}=\mu \delta^{2}=\delta^{4}
$$

Recalling (3.20), we can see now that

$$
\delta=\varepsilon^{1 / 2}, \quad \mu=\varepsilon, \quad \beta=\varepsilon^{1 / 2},
$$

and so all the small parameters are now determined in terms of the governing small parameter $\varepsilon$. Next, we consider the dynamic boundary condition (2.17). Here, our key physical assumption is that the flow is stagnant to leading order in the recirculation zone. Our arguments which produce this conclusion are presented in $\S 3.3$. Now, we note that (2.17) implies that, to leading order,

$$
B= \pm \frac{1}{4} A_{*} \lambda^{(0)^{2}} \frac{\delta^{2}}{\mu} f^{2}
$$

Note that although $\delta^{2}=\mu$, we retain this ratio, and also the ratio $\mu^{2} / \varepsilon^{2}$ as a useful measure of the supercriticality of the wave.

The two expressions (3.23) and (3.26) have now determined $\phi^{(1)}(z, 0)$ in terms of $B$, and so the result can be substituted into (3.22) to yield the final equation for $B$,

$$
\mp B_{z z}+\Delta A_{*}+M\left(A_{*}\right) \pm v B^{2}=0,
$$

where

$$
v=\frac{1}{I\left|A_{*}\right|} \frac{\mu^{2}}{\varepsilon^{2}}
$$


Here, we recall that the upper (lower) signs correspond to $A_{*}>0(<0)$. Here, $v$ is the supercriticality parameter, which is of order unity in general since $\mu=\varepsilon$, and has the same sign as $A_{*}$, and hence the same sign as $B$. Note that, although $\mu=\varepsilon$, here we find it useful to retain them explicitly in $(3.27 b)$ for later use reverting to dimensional variables. The solutions of $(3.27 a)$ must be matched to those of $(3.8 a)$ in the outer zone. The matching conditions are

$$
A=A_{*}, \quad \text { so } \quad B=0 \quad \text { at } \quad|z|=z_{0},
$$

and

$$
A_{X}= \pm \mu^{1 / 2} B_{z} \quad \text { at } \quad|z|=z_{0} .
$$

The first of these conditions defines $|z|=z_{0}$ as the place where criticality is reached in the outer solution, whereas the second condition expresses the continuity of $\phi$ to leading order. Note that the continuity of both $\phi$ and $\phi_{x}$ ensures also the continuity of the vorticity from $(2.10 a)$. The solution of the amplitude equations $(3.8 a)$ and $(3.27 a)$ is described in the next section.

\subsection{Solution inside the recirculation zone}

Here, we must revert to the original Bragg-Hawthorne equation (2.4) but with different functional forms for $C(\psi)$ and $G(\psi)$ denoted by $C_{i}(\psi)$ and $G_{i}(\psi)$, respectively (cf. Keller 1995). In the recirculation zone, $r$ is $O(\delta)$ and $\psi$ is $O\left(\delta^{2}\right)$, whereas the circulation $C_{i}$ is readily seen to also be of $O\left(\delta^{2}\right)$. Hence, we introduce the scaled variables

$$
\Psi=\frac{\psi}{\delta^{2}}, \quad s=\frac{\xi}{\delta^{2}}, \quad \hat{C}_{i}(\psi)=\frac{C_{i}(\psi)}{\delta^{2}} .
$$

Then, the Bragg-Hawthorne equation (2.4) becomes

$$
\delta^{4} \Psi_{z z}+4 s \Psi_{s s}=2 \delta^{2} s G_{i}(\hat{\delta} \Psi)-4 \delta^{4} \hat{C}_{i}(\Psi) \hat{C}_{i \varphi}(\Psi) .
$$

This is to be solved with the boundary conditions that

$$
\Psi=0 \quad \text { on } \quad s=0, \quad s=f^{2}(z) .
$$

We now make the crucial assumption that $G_{i}(0)=0$ so that $\Psi$ is $O\left(\delta^{4}\right)$, and so $\psi$ is $O\left(\delta^{6}\right)$, or $O\left(\varepsilon^{3}\right)$, in the recirculation zone. That is, to leading order, the flow is stagnant there. Note that $\psi_{\xi}=\Psi_{s}$ is then $O\left(\varepsilon^{2}\right)$ in the recirculation zone, whereas the result (3.26) is derived at the $O(\varepsilon)$ term from $\psi_{\xi}\left(z, \delta^{2} f^{2}\right)$. It is also pertinent to note here that the Rayleigh stability criterion requires that $C(\psi) C^{\prime}(\psi)(u-c)$ should be positive. It is readily verified that this is satisfied in the region outside the recirculation zone, but may not be satisfied inside the recirculation zone since $u-c$ changes sign there. Our assumption that $G_{i}(0)=0$, implies that $u-c$ is $O\left(\delta^{4}\right)$, and so in effect we are suppressing the applicability of the stability criterion to a higher order in the asymptotic expansion. It should also be noted here that although $C_{i}(\psi) C_{i}^{\prime}(\psi)$ is $O\left(\delta^{2}\right)$ from (3.29), the choice $C_{i}(\psi) \equiv 0$ (Keller 1995) would ensure at least neutral stability in the recirculation zone. Note that the assumption that the recirculation zone is essentially stagnant is consistent with the numerical unsteady calculations by Lopez (1994) and Rusak et al. (1998).

A commonly used alternative to this procedure is to assume that $G_{i}(\psi)$ and $C_{i}(\psi)$ are determined by analytic continuation from $G(\psi)$ and $C(\psi)$. However, as noted above, this would then lead to violation of the Rayleigh stability criterion at the lowest order being considered. Also, as noted by Leibovich (1984), analytic continuation of $C(\psi)$ into the recirculation zone implies a change of sign of the swirl 
velocity, which seems very unlikely on physical grounds. To avoid this reversion of the swirl inside the recirculation zone Leibovich (1984) proposed to define $C \rightarrow-C$ there. However, even with this amendment, the Rayleigh criterion for stability is still violated. Nevertheless, it is useful to record the outcome of this procedure. In this case $G_{i}(0)=G(0)=2 \Omega_{0}^{2} /\left(1-c^{(0)}\right)+O\left(\varepsilon^{2}\right)$ (see $\left.(2.8 b)\right)$, and it is readily found that then $\phi^{(1)}(z, 0)=O(\varepsilon)$. Consequently, the amplitude equation (3.22) is not determined at $O\left(\varepsilon^{2}\right)$. Instead, in this case, it is necessary to go to higher order, and a different balance to (3.25) holds. Instead, we find that $\mu=\beta=\delta^{2}=\varepsilon^{2 / 3}$, and the amplitude equation $(3.27 a)$ is replaced by

$$
\mp B_{z z}+\Delta A_{*}+M\left(A_{*}\right) \mp \hat{v} B^{3}=0,
$$

where

$$
\hat{v}=\frac{8}{3 A_{*}^{2} I} \frac{\mu^{3}}{\varepsilon^{2}} .
$$

The solutions of this equation are readily found in an analogous manner to those of (3.27a) discussed in the next section. However, for the reasons outlined above, we shall not pursue this case further here in any detail.

\section{Solutions of the amplitude equations}

Let us turn next to finding solutions of the amplitude equations (3.8a) and (3.27a), with the matching conditions $(3.28 a)$ and $(3.28 b)$. First, we integrate $(3.8 a)$ once to obtain, in the outer zone, $|X|>X_{0}=\mu^{1 / 2} z_{0}$,

$$
-A_{X}^{2}+\Delta A^{2}+2 \int_{0}^{A} M\left(A^{\prime}\right) \mathrm{d} A^{\prime}=0,
$$

where we have used the condition that $A \rightarrow 0$ as $|X| \rightarrow \infty$. Next, we use the matching conditions $(3.28 a)$ and $(3.28 b)$ to show that

$$
-\mu B_{z}^{2}\left(z_{0}\right)+\Delta A_{*}^{2}+2 \int_{0}^{A_{*}} M\left(A^{\prime}\right) \mathrm{d} A^{\prime}=0 .
$$

With the omission of the $O(\mu)$ term, this expression determines $\Delta$ in terms of $A_{*}$. It is also pertinent here to recall the inequality (3.9), which holds in the outer zone, and may be rewritten in the form

$$
-\frac{1}{\left|J_{0}\left(\lambda^{(0)}\right)\right|}<\frac{A}{A_{*}} \leqslant 1 \text { for }|X| \geqslant \mu^{1 / 2} z_{0},
$$

where the upper equality is, of course, obtained at $|X|=\mu^{1 / 2} z_{0}$.

In the inner zone, we suppose that the solutions are symmetric, so that we can impose the condition that $B_{z}=0$ at $z=0$. Also, we recall that we normalized the parameter $\mu$ by setting $B=1$ at $z=0$, so that $A_{m}=A_{*}+\mu \operatorname{sign} A_{*}$ is the (maximum) amplitude of the wave at $z=0$. One integration of (3.27a) now yields

$$
B_{z}^{2}=R\left(A_{*}\right)(1-B)-\frac{2}{3} v\left(1-B^{3}\right),
$$

where

$$
R(A)=\left\{\frac{4}{A} \int_{0}^{A} M\left(A^{\prime}\right) \mathrm{d} A^{\prime}-2 M(A)\right\} \operatorname{sign} A_{*} .
$$

The general solution of $(4.4 a)$ can be expressed in terms of elliptic functions, or 
implicitly in the form

$$
|z|=\int_{B}^{1}\left\{R\left(A_{*}\right)(1-u)-\frac{2}{3} v\left(1-u^{3}\right)\right\}^{-1 / 2} \mathrm{~d} u,
$$

from which it follows that

$$
z_{0}=\int_{0}^{1}\left\{R\left(A_{*}\right)(1-u)-\frac{2}{3} v\left(1-u^{3}\right)\right\}^{-1 / 2} \mathrm{~d} u .
$$

This relation determines the width $2 z_{0}$ of the recirculation zone in terms of the parameter $v$. Before proceeding we note that in order for $(4.3 a)$ to have solutions in the range $0<B<1$, we must have

$$
2 v<R\left(A_{*}\right) .
$$

Recalling the definition of the parameter $v$ in $(3.27 b)$ we see that the relation (4.7) places an upper bound on the maximum amplitude $\left|A_{m}\right|=\left|A_{*}\right|+\mu$ of the wave. Further, as this maximum amplitude of the recirculation zone is reached, $z_{0} \rightarrow \infty$; that is, the width of the recirculation zone tends to infinity. This result is consistent with the 'semi-infinite' model of vortex breakdown developed by Keller et al. (1985), and Keller (1995). However, our present results show that this 'semi-infinite' model is only a special limiting case. Although, our travelling-wave solutions in an infinite tube, and the min-max standing-wave solutions in a constricted tube discussed in Wang \& Rusak (1997a), both approach to the Keller et al. (1985) critical solution as the amplitude increases, any stability analogy with the unstable min-max solutions does not necessarily hold. They are different solutions, in particular as our upstream boundary condition is different from the inlet boundary condition used in the study of a constricted tube by Wang \& Rusak (1996), which allows for some upstream influence on the inlet radial velocity. Thus, different problems are being addressed, and no conclusion can be made about the stability of the present waves by directly using the results of Wang \& Rusak (1996). Although a stability study is beyond the scope of the present work, we comment in $\S 5$ on stability issues.

With $B(z)$ now determined from (4.4), we can determine the speed parameter $\Delta$ from (4.2) so that

$$
\Delta=F\left(A_{*}\right)+\frac{\mu}{A_{*}^{2}}\left\{R\left(A_{*}\right)-\frac{2}{3} v\right\},
$$

where

$$
F(A)=-\frac{2}{A^{2}} \int_{0}^{A} M\left(A^{\prime}\right) \mathrm{d} A^{\prime}
$$

Then, from (4.7) and (4.8a) we find that

$$
\Delta-F\left(A_{*}\right)>\frac{4 v \mu}{3 A_{*}^{2}}>0 .
$$

Since $F\left(A_{*}\right)$ is just the value of $\Delta$ when $\mu=0$, we see that the presence of the recirculation zone increases/decreases the speed of the wave according to $c^{(0)}>1(<1)$ $\left(A_{*}>0(<0)\right)$, and so these waves are supercritical. Further, from $(4.8 a)$, we see that as $\mu$ increases so does the speed of the wave. Next, we note that in the outer zone, where $|X|>X_{0}$ and $|A|<\left|A_{*}\right|$, a necessary condition for the existence of the solution is that $\Delta-F(A)>0$, and, hence, on using $(4.8 a)$, we see that

$$
F\left(A_{*}\right)-F(A)+\frac{\mu}{A_{*}^{2}}\left\{R\left(A_{*}\right)-\frac{2}{3} v\right\}>0 \quad \text { all } \quad|A|<\left|A_{*}\right| .
$$


Note that since $F^{\prime}(A)=R(A) \operatorname{sign} A_{*} / A^{2}$, the condition (4.7) ensures that (4.10) is certainly satisfied for $A \approx A_{*}$.

It is now of interest to compare this solution with the corresponding solutions without recirculation zones. These can be obtained from (4.1), now supposed valid for all $X$. Let us suppose that the maximum amplitude is $\hat{A}_{m}$, achieved at $X=0$, say. Then it follows that

$$
\hat{\Delta}=F\left(\hat{A}_{m}\right)
$$

where we let the speed parameter for such waves be denoted by $\hat{\Delta}$ rather than $\Delta$. Then, for $\left|\hat{A}_{m}\right| \leqslant\left|A_{*}\right|$, such waves are well-defined, and have no flow reversals (here we naturally assume that $\hat{A}_{m}$ and $A_{*}$ have the same sign). Indeed, as $\hat{A}_{m} \rightarrow A_{*}$, our asymptotic procedure defines a continuation of such waves to waves with recirculation zones. Here, instead let us consider a continuation for $\left|\hat{A}_{m}\right|>\left|A_{*}\right|$, but for waves without recirculation zones. Such solutions, of course, contain flow reversals, but can nevertheless be formally defined. Thus, let $\hat{A}_{m}=A_{*}+\hat{\mu} \operatorname{sign} A^{*}$. Then, for $0<\hat{\mu} \ll 1$, (4.11) shows that

$$
\hat{\Delta}=F\left(A_{*}\right)+\frac{\hat{\mu}}{A_{*}^{2}} R\left(A_{*}\right)+\cdots .
$$

If we now formally identify $\hat{\mu}$ with $\mu$, and hence analogously $\hat{A}_{m}$ with $A_{m}$, we see that

$$
\hat{\Delta}-\Delta=\frac{2 \mu v}{3 A_{*}^{2}}>0 \text {. }
$$

It follows that, for the same maximum amplitude, the waves with recirculation zones travel faster/slower than waves of the same amplitude without recirculation zones, according to $c^{(0)}>1(<1)\left(A_{*}>0(<0)\right)$. Next, we note that a necessary condition for the existence of waves without recirculation zones, is

$$
F\left(\hat{A}_{m}\right)-F(A) \geqslant 0 \text { all }|A| \leqslant\left|\hat{A}_{m}\right| .
$$

This condition is the counterpart of (4.10) for waves with recirculation zones. Evaluation of (4.14) in the limit $A \rightarrow \hat{A}_{m}$, for $\mu \ll 1$, shows that a necessary condition for the existence of waves without recirculation zones is that

$$
R\left(A_{*}\right) \geqslant 0 .
$$

Note that this condition is much less restrictive than its counterpart (4.7) for waves with recirculation zones.

To make further progress, we must consider some special cases where the form of the nonlinear term $M(A)$ is specified. However, first let us note that the structure of the recirculation zone is independent of the details of the outer zone solution, and depends only on the parameter $R\left(A_{*}\right)$. Now, let the nonlinear term $M(A)$ be given by the cubic expression (3.12), from which it follows that

$$
\begin{aligned}
& F(A)=-\alpha_{1}-\frac{2}{3} \alpha_{2} A-\frac{1}{2} \alpha_{3} A^{2}, \\
& R(A)=-\frac{2}{3} \alpha_{2}|A| A-\alpha_{3}|A|^{3} .
\end{aligned}
$$

Recalling the definition of the parameter $v,(3.27 b)$, we now see that the condition (4.7) reduces here to

$$
\frac{\mu^{2}}{I \varepsilon^{2}}<-\frac{1}{3} \alpha_{2} A_{*}^{3}-\frac{1}{2} \alpha_{3} A_{*}^{4} .
$$

Next, the outer solution is given by $(3.13 a)$ and $(3.13 b)$, and combining these expres- 
sions with $(4.8 a)$ we find that

$$
\Delta+\alpha_{1}=\gamma^{2}=-\frac{2}{3} \alpha_{2} A_{*}-\frac{1}{2} \alpha_{3} A_{*}^{2}+\frac{\mu}{A_{*}^{2}}\left\{R\left(A_{*}\right)-\frac{2}{3} v\right\},
$$

where $\gamma^{2}$ is the defining parameter for the outer solution. However, since $\gamma^{2}>0$, the right-hand side of (4.18) must be positive, and it is pertinent to note that the constraints on the sign of magnitude of $A_{*}$ imposed by (4.18) are not sufficient to ensure the validity of (4.17). The implications of this are discussed below. We should also note that the positivity of (4.18) follows directly from the general result (4.10) in the limit $A \rightarrow 0$. Next, we note that the constant $X_{*}$ in $(3.13 a)$ is given by

$$
\left(z_{0}-z_{*}\right)^{2}=\frac{9}{\alpha_{2}^{2} A_{*}^{4} B_{0}^{2}}\left\{R\left(A_{*}\right)-\frac{2}{3} v\right\},
$$

where $B_{0}$ is obtained from (3.13b), so that $B_{0}=1+3 \alpha_{3} A_{*} / 2 \alpha_{2}$, and $X_{*}=\mu^{1 / 2} z_{*}$.

Let us next consider the corresponding waves without recirculation zones. In this case, the waves are defined by $(3.13 a)$ and $(3.13 b)$ for all $X$. The relation (4.11) combined with $(3.13 b)$ then shows that

$$
\begin{aligned}
\hat{\Delta}+\alpha_{1} & =\hat{\gamma}^{2}=-\frac{2}{3} \alpha_{2} \hat{A}_{m}-\frac{1}{2} \alpha_{3} \hat{A}_{m}^{2}, \\
& =-\frac{2}{3} \alpha_{2} A_{*}-\frac{1}{2} \alpha_{3} A_{*}^{2}+\frac{\hat{\mu}}{A_{*}^{2}} R\left(A_{*}\right) .
\end{aligned}
$$

Here, we recall that $\hat{A}_{m}=A_{*}+\hat{\mu} \operatorname{sign} A_{*}$, and $(4.20 b)$ holds when $\hat{\mu} \ll 1$. Here, $\hat{\gamma}$ is the defining parameter for these waves (the counterpart of $\gamma$ for the waves with recirculation zones), and since $\hat{\gamma}^{2}>0$, the right-hand sides of $(4.20 a)$ and $(4.20 b)$ must be positive. Note that the positivity of (4.20) also follows directly from (4.14) in the limit $A \rightarrow 0$. Further, the general condition (4.15) must also hold here. However, as in the case of the waves with recirculation zones, the positivity of (4.20) does not ensure the validity of (4.15).

It is now necessary to consider the cases $\alpha_{3}>0(<0)$ separately. Let us first suppose that $\alpha_{3}>0$, where the domains of existence for solitary waves, both with and without recirculation zones are depicted in figure 3 . In figure 3 , we plot the quantity $\varepsilon^{2} R\left(A_{*}\right)$ as a function of $\mu$, where we recall from (4.16b) that

$$
R\left(A_{*}\right)=-\frac{2}{3} \alpha_{2}\left|A_{*}\right| A_{*}-\alpha_{3}\left|A_{*}\right|^{3} .
$$

Also, we recall from $\S 3$ that we require $\alpha_{2} A_{*}<0$ in this case. Note that because $\alpha_{2}$ and $\alpha_{3}$ are linear expressions in $a_{1}, a_{2}, b_{1}$ and $b_{2}$ (see (3.11)), and $\hat{\kappa}=\kappa / \varepsilon^{2}, \hat{\sigma}=\sigma / \varepsilon^{2}$ (see (3.8)) are defined by the basic inflow conditions of uniform axial velocity, and uniform rotation, respectively, it follows that $\varepsilon^{2} R$ is, in fact, independent of $\varepsilon$, and a function only of the actual inflow conditions.

Formally, waves with amplitudes below the critical value are represented in figure 3 by $\mu<0$. Such waves have no recirculation zones, and exist provided that $\left|\hat{A}_{m}\right|<$ $\left|2 \alpha_{2} / 3 \alpha_{3}\right| ;$ since $\hat{A}_{m}=A_{*}+\mu \operatorname{sign} A_{*}$, we obtain

$$
R\left(A_{*}\right) \geqslant \alpha_{3} A_{*}^{2} \mu,
$$

where, when equality holds, the solitary wave becomes the so-called 'thick' solitary wave, which has infinite width. Note that this condition also formally defines solitary waves without recirculation zones whose amplitudes exceed $\left|A_{*}\right|$ in absolute value, and are defined by $\mu>0$. In contrast, the condition (4.17) for the existence of solitary 

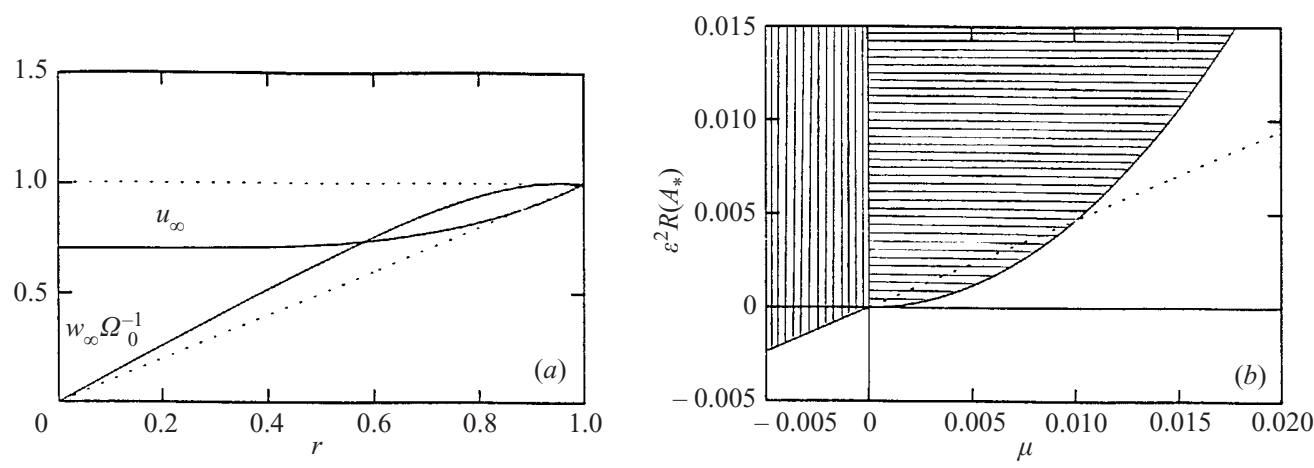

FIGURE 3. Typical domains of existence for solitary waves with and without recirculation zones when $\alpha_{3}>0$. (a) The inflow conditions (solid lines) for the actual values of $\alpha_{2}$ and $\alpha_{3}$ used (the dashed lines show the case of uniform axial velocity and rotation, i.e. $\alpha_{2}=\alpha_{3}=0$ ). (b) The corresponding plot of $\varepsilon^{2} R\left(A_{*}\right)$ as a function of $\mu$; the solid line in $\mu>0$ is for the wave with recirculation zone, whereas the dashed line is for the wave without a recirculation zone. The shaded regions show the domains of existence for waves without recirculation zones when $\mu<0$, and for waves with recirculation zones when $\mu>0$.

waves with recirculation zones can be written as

$$
\varepsilon^{2} R\left(A_{*}\right)>2 \mu^{2} / I\left|A_{*}\right| .
$$

Thus, we see that the family of solitary waves whose amplitudes are below the critical value (i.e. $\mu<0$ ) can be extended to $\mu>0$ as either a family of solitary waves with recirculation zones, or without recirculation zones. The domains of existence for the waves with, or without, recirculation zones do not coincide, and either kind can exist in a region where the other does not. As noted previously, the condition (4.23) places an upper bound on the maximum amplitude $A_{m}=\left|A_{*}\right|+\mu$ of the solitary waves with recirculation zones. If we denote this upper bound by $\mu_{\max }$, then we see that (4.23) implies that $0<\mu<\mu_{\max }$, where

$$
\mu_{\max }^{2}=I\left|A_{*}\right|^{2} \varepsilon^{2}\left(-\frac{1}{3} \alpha_{2} A_{*}-\frac{1}{2} \alpha_{3} A_{*}^{2}\right) .
$$

Note that the right-hand side is a function only of the inflow conditions. As $\mu \rightarrow \mu_{\max }$, the recirculation zone becomes infinitely wide, and reaches a maximum radial extent $\eta_{\max }$, where

$$
\eta_{\max }^{2}=\frac{4 \mu_{\max }}{\left|A_{*}\right| \lambda^{(0)}} .
$$

The maximum radial extent of the recirculation zone is depicted in figure 4 .

To complete this discussion of the case $\alpha_{3}>0$, we show in figure 5 plots of the wave profiles for the upstream conditions of figure 3 , for two representative values of $\mu$. The broadening of the recirculation zone as $\mu$ increases is quite marked. In figure 6 , we show more detail of the shape of the recirculation zone as $\mu$ increases.

Next, we suppose that $\alpha_{3}<0$, and the corresponding plots are shown in figures 7 and 8. In this case, there are two families of solitary waves, one with $\alpha_{2} A_{*}<0$, and the other with $\alpha_{2} A_{*}>0$. If $\alpha_{2} A_{*}<0$, then waves without a recirculation zone exist provided only that (4.20) holds, that is,

$$
R\left(A_{*}\right)>-\frac{1}{2} \alpha_{3}\left|A_{*}\right|^{2}\left(\left|A_{*}\right|-\mu\right) \text {. }
$$

However, if instead $\alpha_{2} A_{*}>0$, then the analogous condition for the existence of waves without a recirculation zone is again (4.26) together with the condition that 


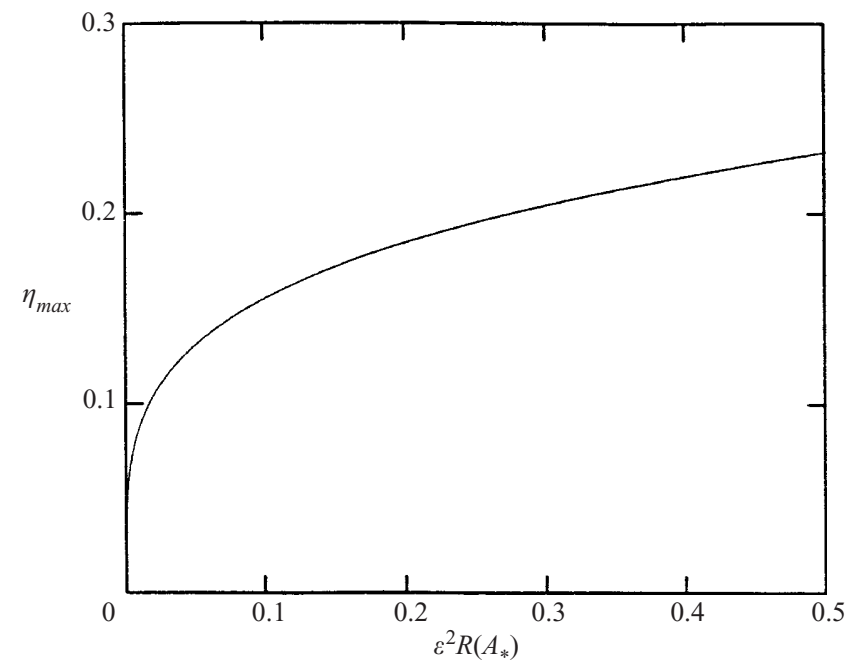

FiguRE 4. The maximum radial extent of the recirculation zone when $\alpha_{3}>0$, as a function of $\varepsilon^{2} R\left(A_{*}\right)$
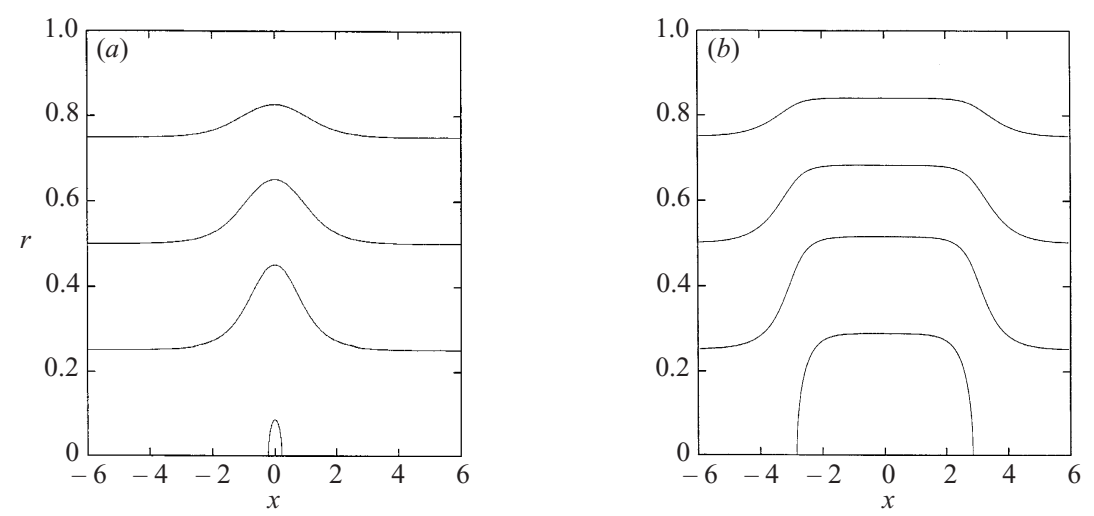

FIGURE 5. Streamline patterns for waves with recirculation zones, when $\alpha_{3}>0$ and for the inflow conditions of figure $3(a)$. (a) $\mu=0.014=0.088 \mu_{\max }$; (b) $\mu=0.159=0.999 \mu_{\max }$.

$\left|\hat{A}_{m}\right|>\left|4 \alpha_{2} / 3 \alpha_{3}\right|$, or

$$
R\left(A_{*}\right)>-\frac{1}{2} \alpha_{3}\left|A_{*}\right|^{3}-\frac{2}{3} \alpha_{2} \mu A_{*} .
$$

However, it can be shown that the conditions (4.26) and (4.27) are equivalent (at least to $O(\mu)$ ), and hence it is sufficient here to use only (4.26).

In contrast, the conditions (4.17) and $\gamma^{2}>0(4.18)$, for the existence of solitary waves with recirculation zones, together give (4.23), and

$$
\varepsilon^{2} R\left(A_{*}\right)>-\varepsilon^{2} \frac{\alpha_{3}}{2}\left|A_{*}\right|^{2}\left(\left|A_{*}\right|-\mu\right)+\frac{2}{3} \mu \frac{\mu^{2}}{I\left|A_{*}\right|^{2}} .
$$

These expressions hold for both families $\alpha_{2} A_{*}<0(>0)$. Here, of course, $\mu>0$, and we see that, after comparing the expression (4.28) with (4.26) (for $\mu \leqslant 0$ ), solitary waves with recirculation zones emerge continuously from the solitary waves without recirculation zones as $\mu$ passes through zero, and $R\left(A_{*}\right)$ passes through $-\frac{1}{2} \alpha_{3}\left|A_{*}\right|^{3}=R_{*}$, say. Again we note that for $\mu>0$, the domains of existence for waves with, or without, 


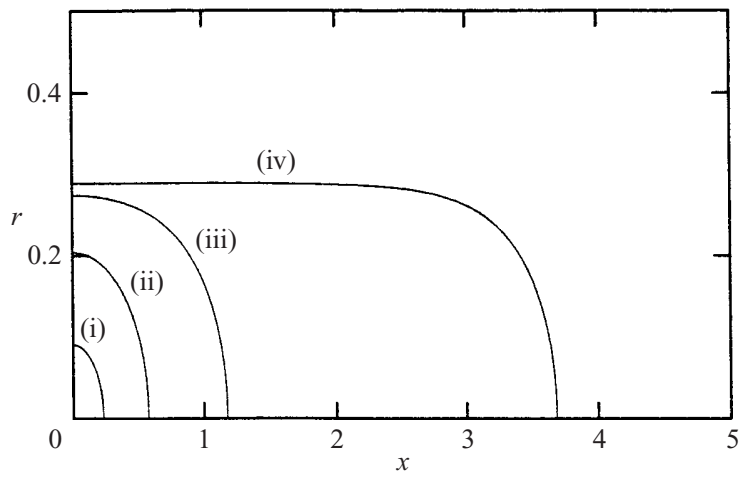

FiguRE 6. The recirculation zone for the case $\alpha_{3}>0$ and the inflow conditions of figure 3(a). (i) $\mu=0.1 \mu_{\max }$, (ii) $0.5 \mu_{\max }$, (iii) $0.9 \mu_{\max }$, (iv) $0.9999 \mu_{\max }$. There is extreme sensitivity as $\mu \rightarrow \mu_{\max }$, and hence this last value is readily distinguished from $\mu_{\max }$.
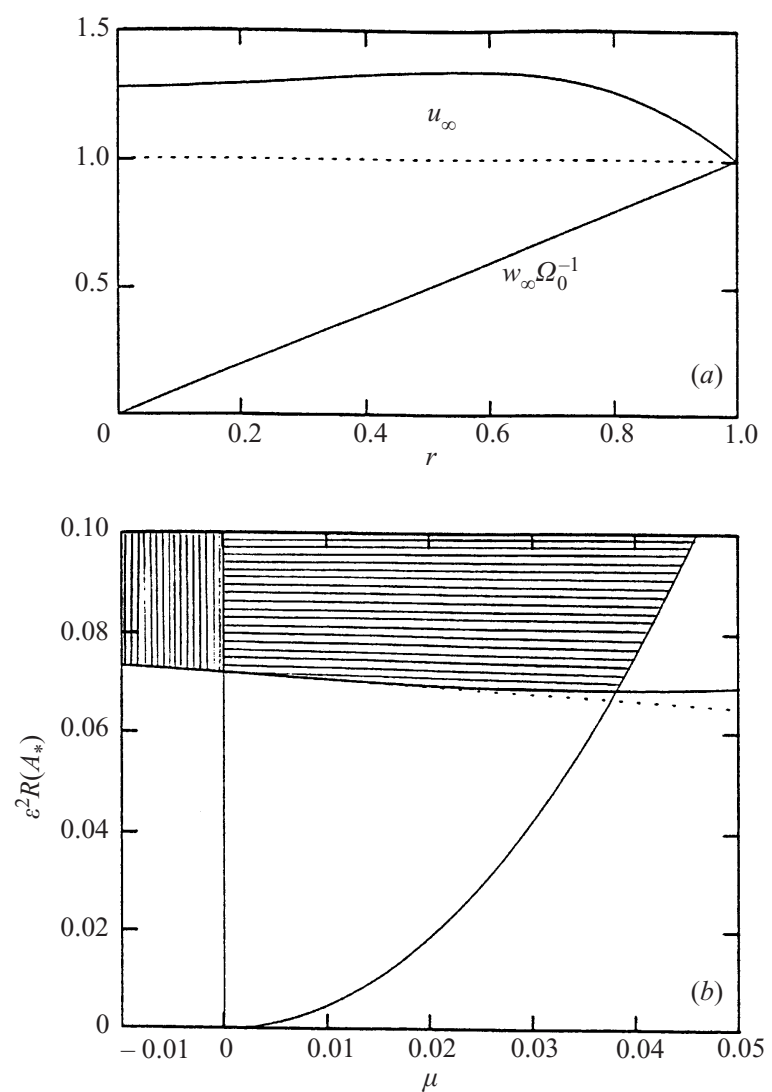

FiguRE 7. Typical domains of existence for solitary waves with and without recirculation zones when $\alpha_{3}<0$. Otherwise, as for figure 3 .

recirculation zones, do not coincide. The criterion (4.28) holds for $0<\mu<\mu_{c}$, where $\mu_{c}$ is defined by the intersection of the boundaries specified by (4.23) and (4.28), so that

$$
\mu_{c}=\left(\frac{1}{2} \varepsilon^{2} R_{*} I\left|A_{*}\right|\right)^{1 / 2}-\frac{1}{6} \varepsilon^{2} R_{*} I+\cdots .
$$



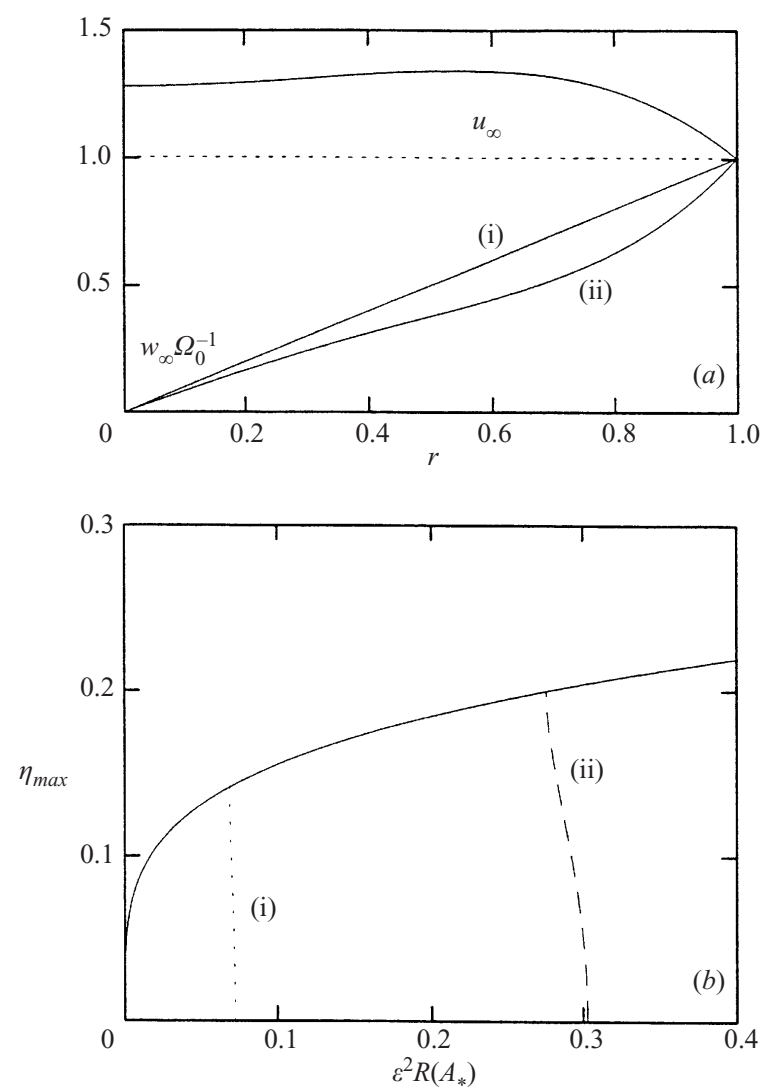

Figure 8. The maximum radial extent of the recirculation zone when $\alpha_{3}<0$, as a function of $\varepsilon^{2} R\left(A_{*}\right)$, for two cases of inflow conditions; on case (i) the angular velocity is uniform, while in case (ii) there is some shear present. The inflow conditions are shown in $(a)$, and note that case (i) is also the inflow condition shown in figure $7(a)$. The maximum radial extent of the recirculation zone is shown in $(b)$, where the solid line is the boundary defined by (4.23), and the dotted and dashed lines are those defined by (4.28) for the case of profiles (i) and (ii), respectively.

For $\mu>\mu_{c}$, the criterion is (4.23). The intersection point $\mu=\mu_{c}$ defines a value of $R\left(A_{*}\right)=R_{c}$, where

$$
R_{c}=R_{*}\left(1-\frac{2}{3} \frac{\mu_{c}}{\left|A_{*}\right|}+\cdots\right) .
$$

Here, we recall that $R_{*}=-\frac{1}{2} \alpha_{3}\left|A_{*}\right|^{3}$, and we note that $R_{c}<R_{*}$. For $0<\mu<\mu_{c}$, equality in the criterion (4.28) determines a limiting wave in which the outer zone solution has infinite width (i.e. $\gamma \rightarrow 0$, in which limit the exponential decay at infinity is replaced by algebraic decay). If we denote the corresponding value of $\mu$ by $\mu_{\min }$, then $\mu_{\min }$ is determined as the smallest real, positive root of the cubic equation

$$
\frac{2}{3} \mu_{\text {min }}^{3}-\varepsilon^{2} R_{*}\left|A_{*}\right| I \mu_{\min }+\varepsilon^{2} I\left|A_{*}\right|^{2}\left(R_{*}-R\left(A_{*}\right)\right)=0 .
$$

Note that here the corresponding value of $R\left(A_{*}\right)$ lies in the range $R_{*}>R\left(A_{*}\right)>R_{c}$. However, for $\mu>\mu_{c}$, it is equality in (4.23) which determines the limiting wave where now it is the recirculation zone which has infinite width. The limiting value $\mu_{\max }$ is now given by (4.24), where of course we now require that $\mu_{\max }>\mu_{c}$ and correspondingly $R\left(A_{*}\right)>R_{c}$. In the range $R_{*}>R\left(A_{*}\right)>R_{c}$, we see that $\mu_{\min }<\mu<\mu_{\max }$, and is an 

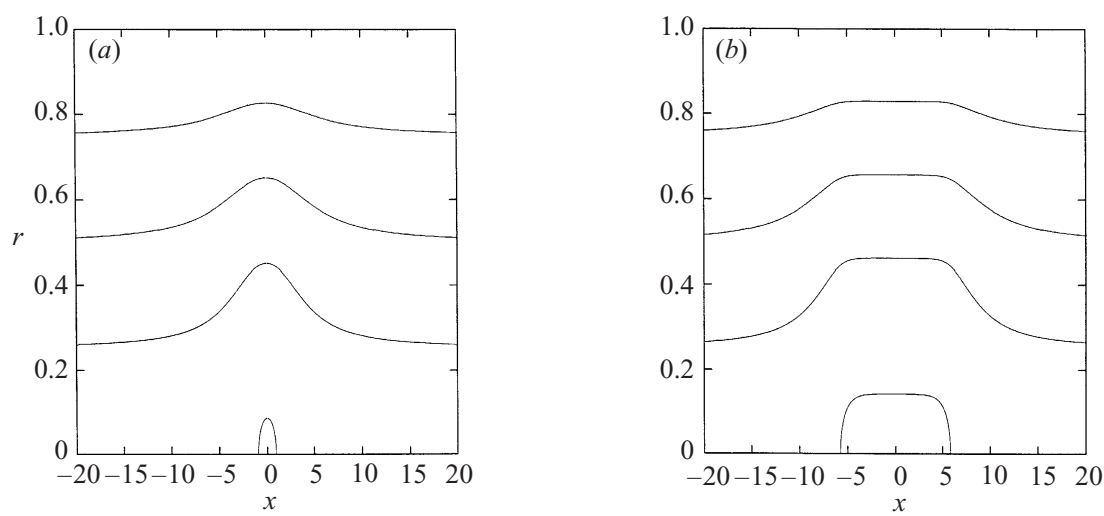

FIGURE 9. Streamline patterns for waves with recirculation zones, when $\alpha_{3}<0$ for the inflow conditions of figure 7(a). (a) $\mu=0.014 \approx \mu_{\min }$; $(b) \mu=0.0385=0.999 \mu_{\max }$.

example of a jump bifurcation; there is no continuation here from waves without a recirculation zone. As $\mu \rightarrow \mu_{\text {min }}$, the recirculation zone reaches a minimum radial extent $\eta_{\min }$, where

$$
\eta_{\text {min }}^{2}=4 \mu_{\text {min }} /\left|A_{*}\right| \lambda^{(0)^{2}} .
$$

In this range $R_{*}>R\left(A_{*}\right)>R_{c}$, it follows that the radial extent of the recirculation zone varies in the range $\eta_{\min }<\eta<\eta_{\max }$. The plot of the variation of the radial extent of the recirculation zone is shown in figure 8 . For $R\left(A_{*}\right)<R_{c}$, there are no steady solitary waves, either with, or without recirculation zones. However, in figure 8 , solitary waves with recirculation zones exist to the right-hand side of the dotted lines in the case of inflow profile (i) and the dashed lines in profile (ii). Note that such solitary waves for inflow profile (i) can exist in a region where there are none for the case of inflow profile (ii).

In figure 9, we show plots of the wave profiles for the upstream conditions of figure 7 , for two representative values of $\mu$; in figure $9(a)$, we show a case when $\mu$ is close to $\mu_{\min }$, where the wave in the outer zone has a very large width (with approximately algebraic decay) whereas in figure $9(b)$, we show a case when $\mu$ is close to $\mu_{\max }$, so that it is now the recirculation zone which has the large width. In figure 10, we show more detail of the shape of the recirculation zone as $\mu$ varies. In figure 11, we compare the wave profiles of two waves with the same speed, but with opposite polarity. The wave of elevation has a recirculation zone as calculated by our present asymptotic model, whereas the wave of depression has no flow reversals and a much lower amplitude (in absolute value). This illustrates the existence of multiple solutions for $\alpha_{3}<0$, and indicates the possibility of a sudden transition from a large-amplitude wave to a small-amplitude wave of large width. In figure 12, we show the profiles of the wave amplitude $A(X)$ for waves with recirculation zones, as predicted by our present asymptotic model and, for comparison, as predicted by the modified KdV solution (3.13a). Note that for $\mu \approx \mu_{\min }$ the difference is barely discernible, but for $\mu \approx \mu_{\max }$ it is clearly seen.

We note that the flow inside the separation bubble is assumed to be stagnant in our model. The modified $\mathrm{KdV}$ solution (3.13), which can be formally extended to amplitudes allowing recirculation, leads to the incorrect results when $\mu \approx \mu_{\max }$ as it implicitly assumes the analytic continuation of the governing equations into the separation zone. This result agrees with the findings of Goldshtik \& Hussain (1997). 


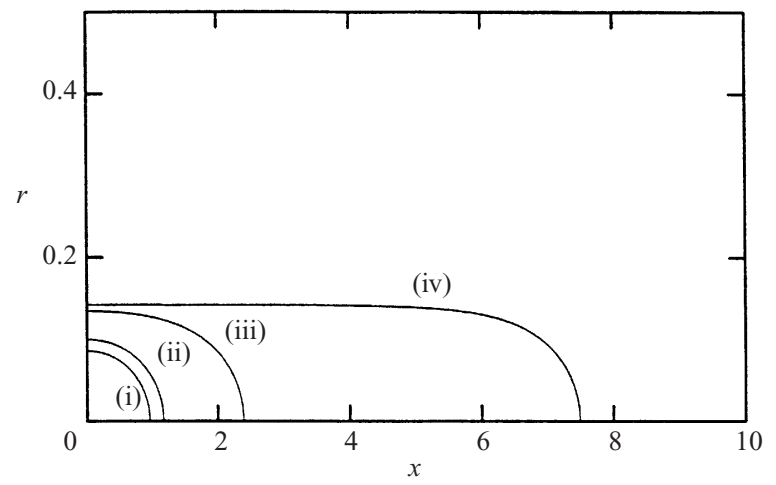

FIGURE 10. The recirculation zone for the case $\alpha_{3}<0$ and the inflow conditions of figure 7(a). (i) $\mu=0.371 \mu_{\max } \approx \mu_{\min }$, (ii) $0.5 \mu_{\max }$, (iii) $0.9 \mu_{\max }$, (iv) $0.9999 \mu_{\max }$.
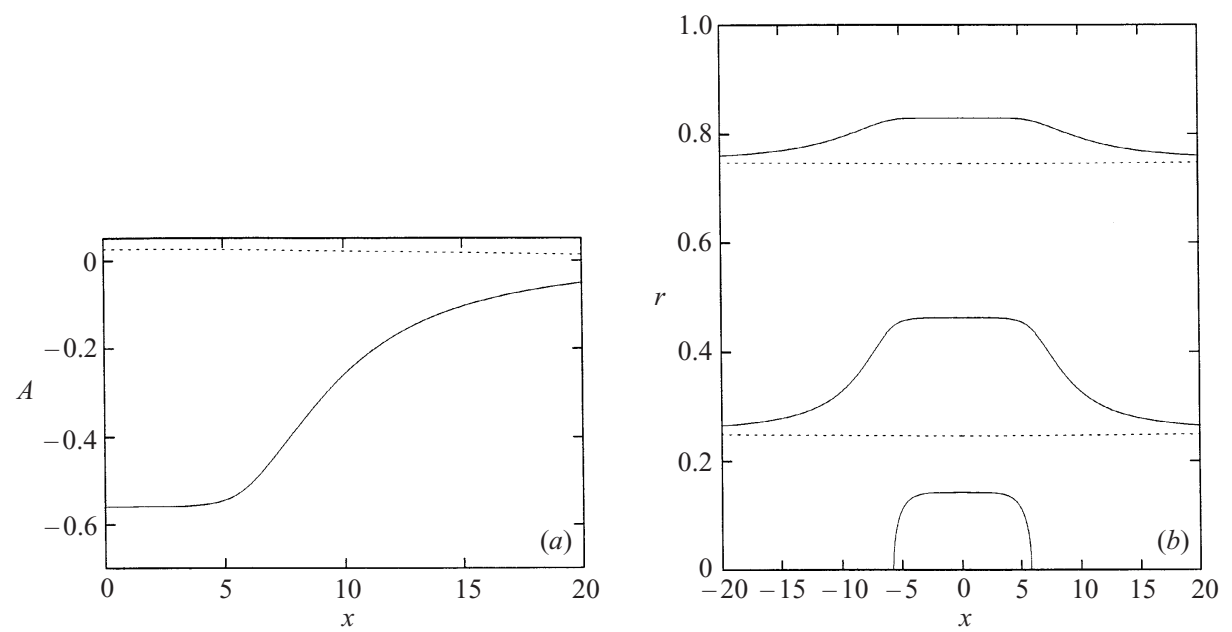

FIGURE 11. Comparison of $(a)$ the amplitude profile $A(X)$ and $(b)$ the streamline patterns for waves with and without recirculation zones (solid and dashed lines, respectively). These respective solitary waves have the same phase speed. The maximum amplitudes are 0.5604 for a wave of elevation (with reversal) and 0.0252 for a wave of depression, respectively.

The stagnant flow in the separation zone was also observed in numerical calculations by Rusak et al. (1998).

Finally, in this section, we consider the special case when $\alpha_{3}=0$, so that $M(A)$ (equation (3.12)) contains only quadratic nonlinearity; note that this requires that $a_{2}=b_{2}=0$ in the expression (3.11) for $U$ and $\Omega$. In this case, the solution (3.13a) in the outer zone reduces to the familiar KdV solitary wave (3.14). The continuation to waves with recirculation zones now requires that $\alpha_{2} A_{*}<0$, and occurs continuously as $\mu$ passes through zero. Here $R\left(A_{*}\right)=-\frac{2}{3} \alpha_{2}\left|A_{*}\right| A_{*}$, and the domain of existence for solitary waves with recirculation zones is (4.23) with $\alpha_{3}=0$, that is

$$
-\frac{1}{3} \varepsilon^{2} \alpha_{2} A_{*}^{3} I>\mu^{2}
$$

In contrast, the only requirement for the existence of solitary waves without recirculation zones is that $\alpha_{2} A_{*}<0$, for both $\mu>0$ and $\mu<0$.

It is also of interest to consider the special case when $R(A) \equiv 0$, so that $M(A)$ is a linear function of $A$, and can be absorbed into the detuning parameter $\Delta$. This case 


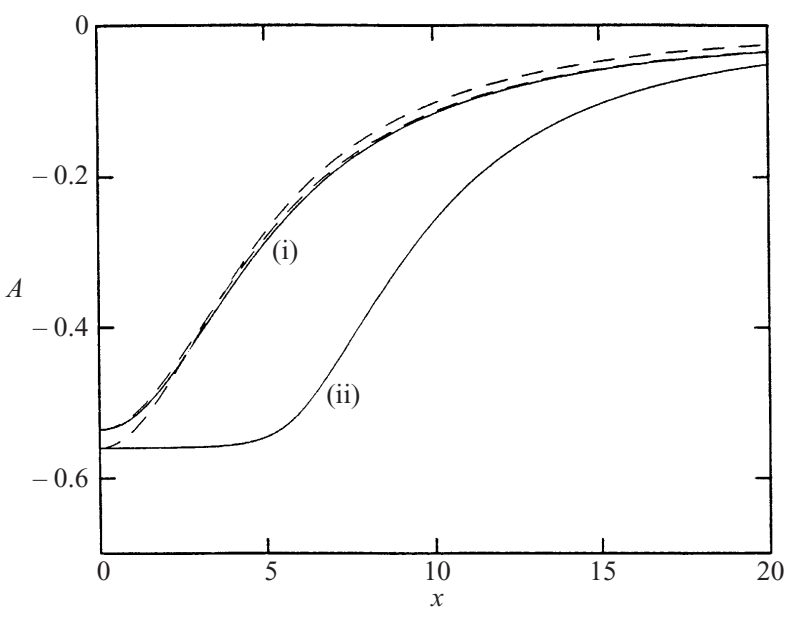

FIGURE 12. Comparison of the wave amplitude profile $A(X)$ for waves with a recirculation zone, as predicted from our model (3.8), (3.27), (3.28) and that predicted by (3.13a) (solid and dashed lines, respectively) when $\alpha_{3}<0$ with the inflow conditions of figure $7(a)$. Here, case (i) is for $\mu=0.364 \mu_{\max }=0.014$ where the difference between these predictions is barely visible, whereas case (ii) is for $\mu=0.999 \mu_{\max }=0.0385$ where the difference is clearly visible.

corresponds, of course, to the situation when the inflow conditions are just those of uniform axial velocity and uniform angular velocity. Then it is well-known (Fraenkel 1964) that there are no solitary waves without recirculation zones, and our analysis shows also, from (4.7), that there can be no solitary waves with recirculation zones.

\section{Discussion}

In the preceding sections we have described an asymptotic construction of solitary waves with recirculation zones located on the tube axis, for the situation when the inflow conditions upstream are close to, but slightly different from uniform axial flow and uniform rotation. Although our procedure can, in principle, be applied to quite general, albeit small, departures from this state of uniform inflow conditions, our analysis shows that the presence of such solitary waves is sensitive to the actual upstream axial and rotational shear configurations. However, the structure of the inner and recirculation zones is universal, and depends on the inflow conditions through the single constant $R\left(A_{*}\right)$ (see $(4.3 b)$ ). In particular, in all cases, the recirculation zone has a maximum amplitude, given by (4.6), and as this maximum amplitude is approached, the recirculation zone increases in width. From a detailed analysis of the special case, when these shear profiles are described by (3.11), we can infer that there are two possible scenarios. In the first, deduced from the case $\alpha_{3}>0$ in $\S 3$, there is a continuous transition as the solitary-wave amplitude is increased through the critical amplitude to a wave with a recirculation zone. In the second, deduced from the case $\alpha_{3}<0$ in $\S 3$, although there may again be a continuous transition, there also exists a range of parameters characterizing the inflow conditions where there is a jump bifurcation; that is, solitary waves with recirculation zones can exist in such a parameter range without any continuation from the low-amplitude solitary waves. Note that although our asymptotic procedure can be readily extended to higher order, thus alleviating to some extent the present restriction to inflow conditions which are only a small departure from the uniform state, we contend that the dominant effects 

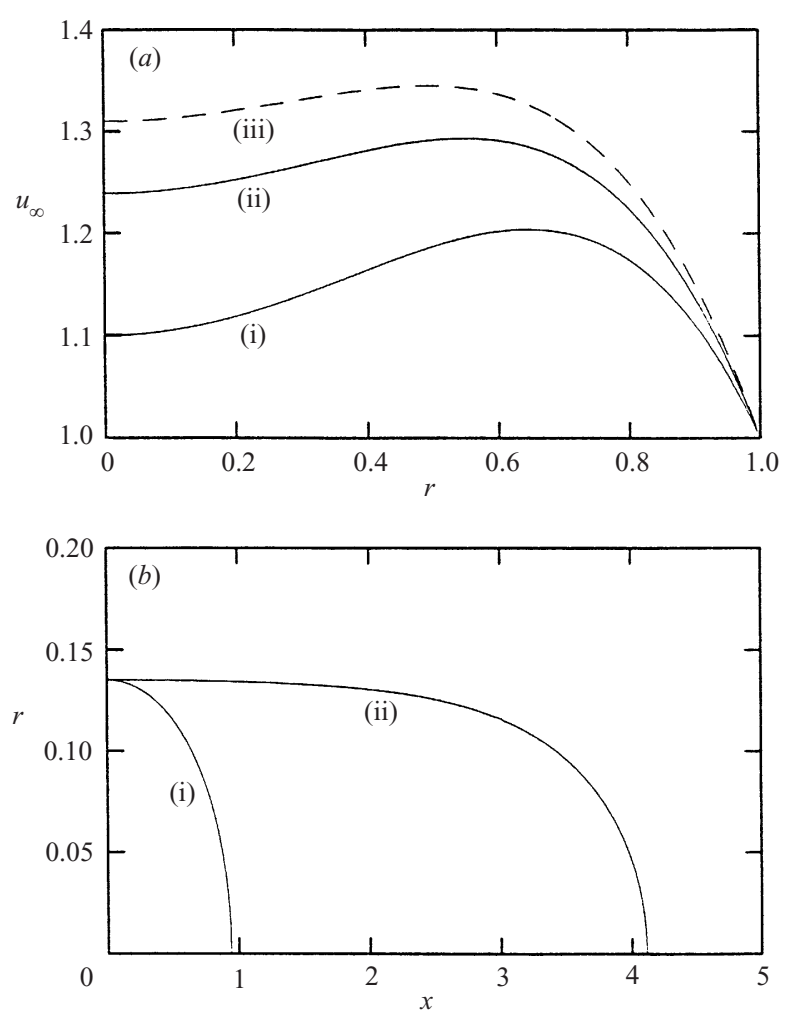

FIGURE 13. (a) Jetlike profiles of the inflow axial velocity, while the angular rotation is uniform. (b) The recirculation zone. $\mu=0.035, \eta_{\max }=0.1855, \eta_{\min }=0$ corresponds to profile (i), $\eta_{\max }=0.136$, $\eta_{\min }=0.112$ corresponds to profile (ii). There is no solution with a recirculation zone for the case of profile (iii).

of variable inflow profiles are captured in the present model. Even in the framework of the present formulation, we can consider more complicated velocity profiles by using polynomial approximations of these profiles. A polynomial description of the upstream axial and swirl velocity profiles of the $N$ th order in $r^{2}$ will lead to a polynomial nonlinearity of $(N+1)$ th order in the resulting equation for the wave amplitude.

Here, we will use the illustrative models discussed in $\S 4$ to demonstrate explicitly how variations of the axial and azimuthal velocities in the approaching vortex affect the properties of the solitary waves with recirculation zones. Figure 13 shows that for a given uniform swirl and a jet-like axial velocity, as the magnitude of the axial velocity increases, the region of existence of the solitary wave with a recirculation zone shrinks. Note that, in figures 13 and 14, we have set rather large deviations $(0.2-0.3)$ from the uniform flow. In our experience, asymptotic models may provide good results even when the small parameter is not so small. Figures 13 and 14 illustrate what kind of approaching vortex might produce a separation 'bubble' of about $0.2-0.3$ of the radius of the tube, a size which is typical in experiments. Namely, for a small jet magnitude (especially with some deficit near the axis) there is a unique solitary wave with recirculation. As we increase the magnitude of the axial velocity, there exist multiple wave solutions and the flow may jump between them; also, there is a minimum size of the recirculation zone. When we further increase 

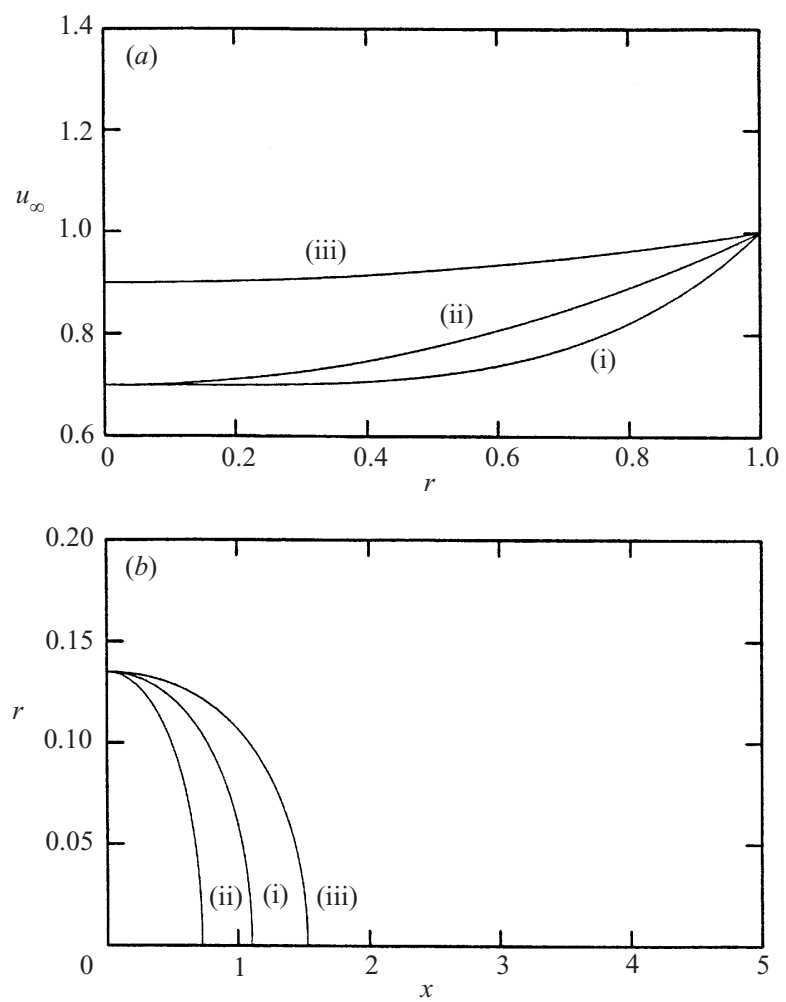

FIGURE 14. (a) Wakelike profiles of the inflow axial velocity, while the angular rotation is uniform. (b) The recirculation zone. $\mu=0.035$; (i) $\eta_{\max }=0.1743$, (ii) 0.2065 , (iii) 0.1569 , and $\eta_{\min }=0$ for all cases.

the axial velocity, there is no solitary wave solution with a recirculation zone. For a wakelike axial velocity (figure 14) in the approaching vortex, the transition from a wave without a recirculation zone to the wave containing a recirculation zone is continuous. For smaller deficits and wider profiles of the axial wakelike velocities, the recirculation zone becomes wider with the same radial extent and the value of the maximum extent becomes smaller. We note that for the jetlike axial profile the effect is opposite, i.e. for stronger and narrower profiles of the axial velocity, the maximum extent of the recirculation zone becomes smaller. This result appears to contradict that obtained by Keller, Egli \& Althaus (1988) for the particular case of a semi-infinite recirculation zone; however, the method of construction used by Keller et al. (1988) for the recirculation zone requires the swirl magnitude to decrease as the strength of the axial jet is increased. In fact, as we demonstrate in the next paragraph, the size of the recirculation zone can be expected to increase with the swirl magnitude, and we suggest that it is this effect which determined the result obtained by Keller et al. (1988).

In figures 15 and 16 we show how different profiles of the swirl velocity affect the properties of the solitary wave with a recirculation zone. It is clearly seen that for a sufficiently wide jetlike, constant or wakelike profile of the axial velocity $\eta_{\max }$ increases monotonically as the magnitude of the swirl increases. In turn, for narrow jetlike profiles of the axial velocity, the solitary waves with a recirculation zone exist only for sufficiently large swirls (to the right of curve (ii), figure 15). This is a typical 


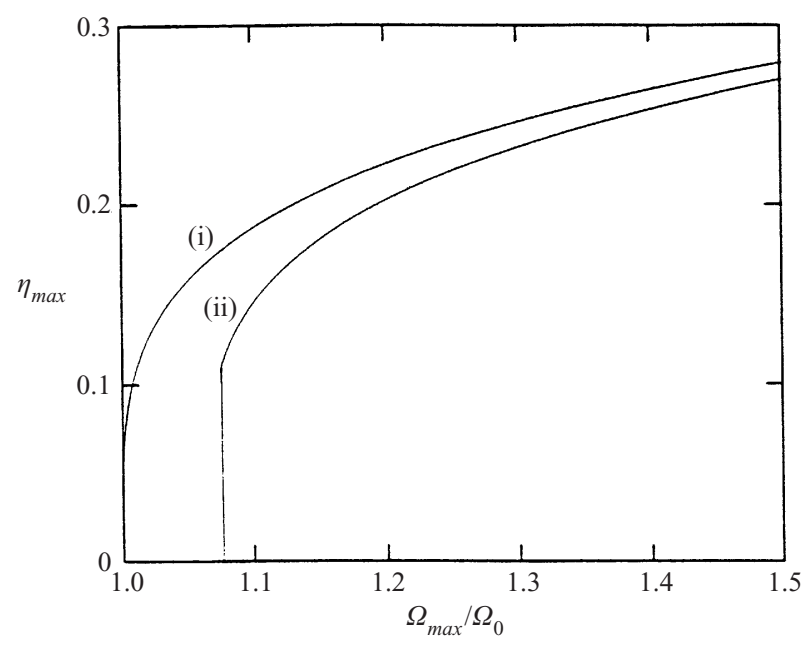

FIGURE 15. The maximum radial size of the recirculation zone, $\eta_{\max }$, as a function of the maximum swirl $\Omega_{\max }$ (i.e. the maximum value of $w_{\infty} / r$ in $(2.6 b)$ ). Curve (i) corresponds to uniform axial velocity $u_{\infty}=1$, and curve (ii) is for the jetlike profile $u_{\infty}=1+0.25\left(1-r_{2}\right)$.
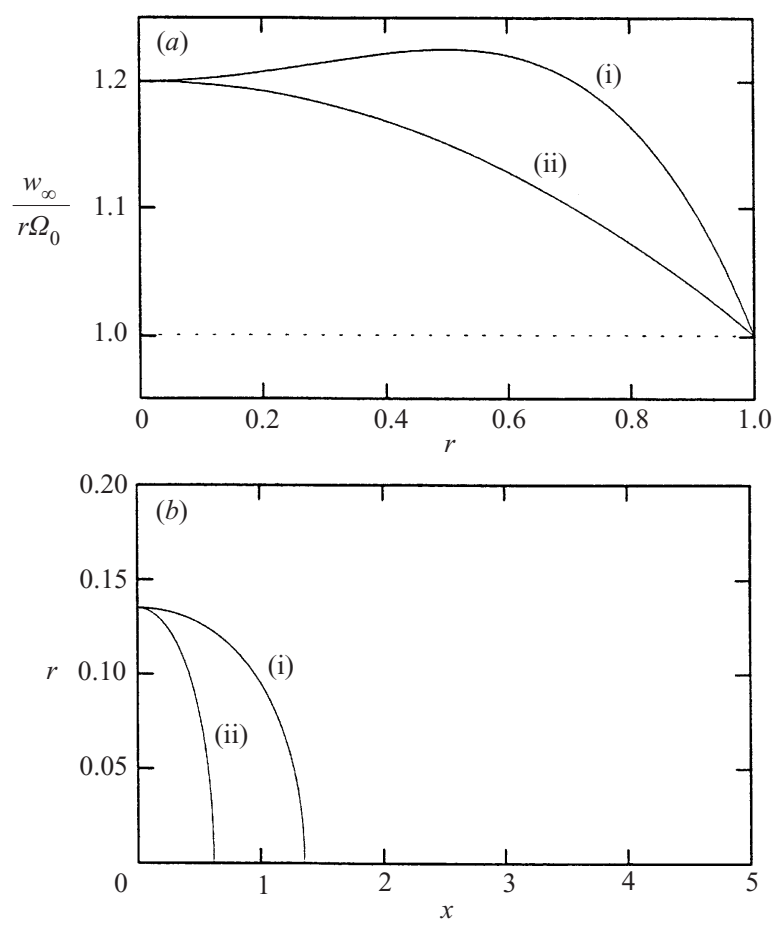

FIGURE 16. (a) Inflow profiles of the swirl, while the axial velocity is uniform, $u_{\infty}=1$. (b) The recirculation zone. $\mu=0.035$, and $\eta_{\max }=0.1626,0.2219$ for the profiles (i) and (ii), respectively.

case for the experiments of Leibovich (1991) where the 'bubble' type of breakdown was not observed for small swirls. In figure 16, we show that wider profiles of the swirl results in a smaller maximum amplitude of the recirculation zone. If the radial extent of the recirculation zone is the same, the flow with a wider profile of swirl, for a given profile of the axial velocity, produces a wider recirculation zone. 
It is pertinent to compare our results with those of others, such as Leibovich and collaborators (see, for instance, Leibovich 1970, 1991; Leibovich \& Randall 1973; Leibovich \& Kribus 1990), who have used long-wave theories to study vortex breakdown. We note here two crucial differences. First, weakly nonlinear theories, which are appropriate when the inflow conditions contain substantial axial and/or rotational shear, usually lead to the KdV equation, or occasionally, the modified KdV equation. Although such theories can provide useful information on vortex breakdown in some circumstances, they cannot be used beyond their range of validity of small amplitudes, and, in particular, cannot be used to describe incipient flow reversal. The exceptional case is precisely that studied here, namely, when the inflow conditions are only weakly sheared and close to uniform axial velocity and uniform rotation; as we have shown, in this special case, a theory can be developed for finite-amplitude long waves. Secondly, as we have already noted, the commonly used practice of determining the circulation and vorticity in the recirculation zone by analytic continuation, does not lead to physically meaningful solutions (see, for instance, Keller et al. 1985; Keller 1995). In $\S 3.3$, we described briefly the implementation of this hypothesis, and the outcome is (3.32), in place of (3.27). However, although analogous solutions to those described in $\S 4$ can be readily constructed, they are very likely to be unstable by Rayleigh's criterion, as we have already noted in $\S 3.3$. Further, we note that the nonlinear term in $(3.32 a)$ has the opposite polarity to that in $(3.27 a)$. This has the consequence that the necessary condition (4.6) for the existence of solutions in the inner zone is now replaced with $R\left(A_{*}\right)+2 v>0$. This is a very mild constraint, and, in particular, can be satisfied when $R\left(A_{*}\right)=0$, which is the case when the inflow conditions are uniform axial velocity and uniform rotation. Thus, the use of (3.32a) leads to the incorrect result that uniform inflow conditions can allow for a solitary wave with a recirculation zone, in marked contrast to numerous theoretical and numerical studies (see, for instance, Fraenkel 1964; Keller et al. 1985; Hanazaki 1996), and the numerical experiments of Kopecky \& Torrance (1973). Note that although Hanazaki (1996), in numerical simulations of the full Navier-Stokes equations for the case of uniform inflow conditions, reported the formation of recirculation zones on the axis of the tube, the recirculation zones were found in a train of waves downstream of a local contraction in the tube wall.

A systematic stability analysis of the stationary solutions obtained by us is beyond the scope of this study. However, we would like to present some speculations that our solitary-wave solutions may be stable in an axisymmetric framework. Numerical simulations of the unsteady Euler equations for a similar problem for internal waves in a stratified fluid have shown that solitary waves containing a separation zone are stable (Aigner et al. 1999). Further, such waves are often observed in the atmosphere and oceans that also support the assumption of their stability (see Derzho \& Grimshaw 1997 for the relevant references). We expect that the situation in the rotating fluid may be similar, owing to the close similarity of the governing equations. Indeed, some preliminary simulations by Aigner (2001) indicate that these inertial solitary waves with recirculation zones found here are very robust. Nevertheless, some other numerical simulations of solitary-like disturbances in rotating fluids do indicate that these disturbances can be unstable, even in an axisymmetric formulation (Lopez 1994; Wang \& Rusak 1997a; Rusak et al. 1998). In Lopez (1994), the breakdown of the initial vortex was induced by a local contraction of the tube; thus, at high Reynolds number, two stable branches of solutions are part of one branch with a fold, with the part of the branch in the fold being unstable. The two stable solutions correspond to the slightly disturbed (by the contraction) initial flow, and to a nonlinear lee 
train, accordingly. Unstable solutions consist of accelerating (flow over contraction) and retarding parts (Beran \& Culick 1992). In the ideal case, these parts integrally compensate each other and create a zero-mass solution that is consistent with the boundary conditions at the inlet. Our formulation for an infinite tube does not allow such solutions and therefore any stability analogy with our solutions is not relevant. Further, we note that Brown \& Lopez (1990) examined vortex breakdown in a straight tube caused by viscous dissipation; however, this case is beyond the scope of the present study. Wang \& Rusak (1997) and Rusak et al. (1998) described another kind of unstable solitary-like disturbance (their 'min-max' solutions) for the case of a constricted tube. However, since the inflow boundary conditions of Rusak et al. (1998) do not coincide with our boundary conditions at far upstream for any finite length of the tube, their stability analysis for their 'min-max' solutions does not apply to our solutions.

Rusak and collaborators (see for instance, Rusak et al. 1998) have also proposed a model for a near-critical swirling flow, but again for the case of a constricted tube. They studied the effects associated with small-inlet azimuthal vorticity perturbations in a straight tube (Rusak 1998), or with small pipe divergence, or due to slight viscosity (see the references in Rusak et al. 1998). It was shown that a regular asymptotic expansion, in terms of a small departure from the initial flow due to these perturbations, is singular around the critical swirl. Thus, relatively large disturbances (of the order of the square root of the perturbation) may be induced by small perturbations when the approaching flow is near-critical. The same result is obtained in our study. Rusak (1998) deals with a relatively general profile of axial and swirling flows. However, the perturbation due to the induced disturbance in the model of Rusak (1998) should still be small compared with the flow field in the incoming columnar vortex. Thus, this model is not set up to give a description of disturbances, which are large in comparison with the incoming flow. Nevertheless, Wang \& Rusak (1997b) accounted for the effect of weak viscosity, and then obtained a good agreement of their asymptotic model with the numerical simulations of Beran \& Culick (1992) for the dependence of the minimum axial velocity (on the axis) as a function of swirl, although comparisons of the wave profiles were not presented. On the other hand, our model is constructed to describe such large disturbances, which may lead to the formation of a separation 'bubble', although the form of the incoming flow in our study is more restricted. We also note that if small-amplitude models, such as those of Wang \& Rusak (1997b) and Rusak (1998), are nevertheless applied to describe large disturbances containing separation zones, there is an inevitable, implicit assumption of the analytic continuation of the solution from outside to the inside of the separation zone. On the other hand, the numerical simulations, such as those of Wang \& Rusak (1997a), show that a stagnation zone naturally appears as a result of unsteady evolution.

Next, we observe that although our emphasis in this paper is on solitary waves with recirculation zones, our asymptotic model can be readily adapted to describe conjugate columnar states and periodic wavetrains as well. Consider first the issue of columnar states, where we turn to (3.8a) for the wave amplitude in the outer zone. Putting $A_{X X}=0$ we see that the resulting nonlinear equation, $\Delta A+M(A)=0$, has, in general, a multiple set of solutions for the constant amplitude $A$. Thus, if $M(A)$ is a polynomial of the $N$ th order, there may be up to $N$ such conjugate states, one of these being $A=0$. For instance, the cubic form (3.12) for $M(A)$ will yield three conjugate states if $\alpha_{2}^{2}>4 \alpha_{3}\left(\Delta+\alpha_{1}\right)$, and only one otherwise. When $\alpha_{3}=0$ there are always two real conjugate states. 
Periodic wavetrain solutions can now be superimposed on each conjugate state. In the outer zone, such periodic wavetrains still satisfy $(3.8 a)$ in the outer zone, whereas here we confine our attention to one spatial period of the wave, and with this constraint, figure 2 again defines the problem configuration. Now, one integration of $(3.8 a)$ will yield (4.1) again, but with an arbitrary constant on the right-hand side in place of zero. In the inner zone, the governing equation is again (3.27), and one integration will yield an equation identical in form to (4.3a), but with a different definition of $R\left(A_{*}\right)$. For a periodic wave, we suppose that the amplitude varies between $A_{m}=A_{*}+\mu \operatorname{sign} A_{*}$ and $A_{0}$ (for a solitary wave $A_{0}=0$ ), and then the arbitrary constant described above is readily determined in terms of $A_{0}$. We then find that, in place of $(4.3 b)$, for a periodic wavetrain

$$
R(A)=\left\{\frac{A \int_{A_{0}}^{A} M\left(A^{\prime}\right) \mathrm{d} A^{\prime}}{A^{2}-A_{0}^{2}}-2 M(A)\right\} \operatorname{sign}\left(A_{*}\right) .
$$

With this new definition of $R\left(A_{*}\right),(4.3 a)$ determines $B(z)$ as before, and in particular (4.5) and (4.6) continue to hold. The speed parameter $\Delta$ is now given by

$$
\Delta=F\left(A_{*}\right)+\frac{\mu}{A_{*}^{2}-A_{0}^{2}} R\left(A_{*}\right)-\frac{2}{3} v,
$$

where

$$
F(A)=-\frac{2}{A^{2}-A_{0}^{2}} \int_{A_{0}}^{A} M\left(A^{\prime}\right) \mathrm{d} A^{\prime},
$$

which replace the expressions $(4.8 a)$ and $(4.8 b)$, respectively. Note that $F^{\prime}(A)\left(A^{2}-\right.$ $\left.A_{0}^{2}\right)=R(A) \operatorname{sign} A_{*}$. The necessary condition (4.6) for the existence of solitary waves with a recirculation zone holds here too for periodic waves. It thus follows from (5.2a) that, for the usual case when $\left|A_{*}\right|>\left|A_{0}\right|$, these periodic waves, like their solitary wave limit, are supercritical, and as $\mu$ increases, so does the speed. The counterpart of (4.10) is the necessary condition for the existence of these periodic waves, $\left(A^{2}-A_{0}^{2}\right)(\Delta-F(A))>0$, or using $(5.2 a)$

$$
\left(A^{2}-A_{0}^{2}\right)\left\{F\left(A_{*}\right)-F(A)+\frac{\mu}{A_{*}^{2}-A_{0}^{2}} R\left(A_{*}\right)-\frac{2}{3} v\right\}>0 .
$$

which should hold for all $A$ between $A_{0}$ and $A_{*}$. As for the solitary wave case, this is certainly satisfied when $A \approx A_{*}$, but does provide a possible constraint when $A \approx A_{0}$. Note also that while solitary waves with recirculation zones depend on the single parameter $\mu$ ( or $A_{m}$ ) for given inflow conditions, these periodic wavetrains depend on two parameters $\mu$ and $A_{0}$; in particular, the wavelength is then determined. Clearly, as $A_{0} \rightarrow 0$, the wavelength tends to infinity and we recover the solitary wave. Further, note that if instead $\left|A_{0}\right|>\left|A_{*}\right|$, then (5.2a) predicts the existence of subcritical periodic wavetrains with recirculation zones.

It is pertinent to note here that, although our discussion in the preceding paragraph demonstrates the existence of periodic wavetrains with recirculation zones, technically our derivation of the outer zone amplitude equation ( $3.8 a$ ) requires that we can specify the inflow conditions at infinity. Clearly, this is not possible for a periodic wavetrain, and indeed, this is a well-known feature of steady nonlinear wave equations, such as that considered here. Instead, we can interpret the present construction of a periodic wavetrain in one of two ways. Either, we can say that it is a modulated periodic wavetrain which is locally valid as an asymptotic approximation in some region of $X$ space, but must be matched to the correct conditions at infinity by other appropriate 
solutions, or, we can say that it represents a valid (asymptotic) periodic solution to the steady-state equations, whose mean-value after spatial averaging consists of a nearly uniform axial velocity and angular velocity, together with a superimposed mean state $\langle A\rangle W(\xi)$, where $\langle A\rangle$ is the mean-value of $A$. In this latter case, it is not possible to make a simple identification of the shear components $U$ and $\Omega$, in (2.7) and (2.8), respectively, with this mean state, which in any case, is not a solution of the governing equation in general. We shall not pursue this topic of periodic wavetrains any further here, as we intend to discuss them in more detail elsewhere.

Although our primary purpose in this paper is to demonstrate the construction of solitary waves with recirculation zones, it is relevant to make some remarks about the relationship of these present results to the phenomenon of vortex breakdown. It is widely accepted that the 'bubble' appearing at the axisymmetric stage of vortex breakdown is the manifestation of the incipient breaking of a large-amplitude inertial wave (see, for instance, Keller et al. 1985; Keller 1995; Leibovich 1984; Leibovich \& Kribus 1990). The mechanisms which may lead to such wave-breaking are usually associated with flow divergence, or perturbations in the inflow azimuthal vorticity. These transient processes are beyond the scope of this present work, where we have examined the steady-state structure of such recirculation zones, or 'bubbles'. We note that many experimental observations show that the development of a 'bubble' is associated with the formation of a stagnation point (Sarpkaya 1971), an initial expansion of the recirculation zone, followed by a contraction, with the occurrence of a second stagnation point. This tendency towards a steady-state structure is quite consistent with the results obtained here. The precise nature of the transient processes which lead to the contraction of the 'bubble' to a steady state would seem to be unclear at the present time. One possibility is effectively dissipative (from the point-of-view of the axisymmetric system), involving the transfer of energy from the axisymmetric wave to non-axisymmetric disturbances owing to a three-dimensional instability as proposed by Leibovich (1984) (see also Leibovich \& Randall 1973; Leibovich 1991). Escudier (1988), and Keller et al. (1985) argued that the 'bubble' formation consists of a two-stage transition. The first (upstream) is a loss-free and force-free transition from the approaching vortex and leads to a semi-infinite columnar flow with stagnation zone near the centreline. The second transition is similar to an internal hydraulic jump and is essentially dissipative. Those two transitions may combine to a single dissipative transition if their propagation speeds are close and therefore create a 'bubble' -like structure. Another possibility is that the 'bubble' formation is essentially inertial and non-dissipative. This point-of-view is supported by our results in-so-far as we have demonstrated that the presence of the 'bubble' zone in an infinite tube is related to the global structure of the inertial wave, and its existence is sensitive to the inflow conditions. On the other hand, it must be conceded that our results also show that the structure of the 'bubble' zone is universal, and this could be interpreted as being due to a redetermination of the circulation and vorticity inside the 'bubble' by dissipative processes.

The effects due to instabilities and dissipation are also very important near the downstream stagnation point, where their combination may lead to the formation of three-dimensional spiral structures and turbulence. It is not our intention here to discuss the mechanisms leading to these various kinds of breakdown. However, we note that our model can explain the observation (see Leibovich 1991) that for a jetlike profile of axial velocity, the 'bubble' type of breakdown occurs only for sufficiently large inflow swirls. The margin between swirls such that the 'bubble' does or does not exist is quite sharp. This may explain why the 'bubble' type of 
breakdown may suddenly appear and then disappear (replaced with a spiral-type of breakdown) even when the inflow varies only slightly. Finally, we again recall that our formulation is not directly related to the existing experimental and numerical studies of vortex breakdown as we formulate our problem in an infinite straight tube. Our main result is a construction of the asymptotic procedure for the description of large-amplitude inertial solitary waves with recirculation zones. This result is a first step to more realistic formulations. Our procedure can be applied to the more realistic case of slightly uneven constricted tube with fixed zero radial velocity at the inlet as considered by Lopez (1994) and Beran \& Culick (1992), or to the case of fixed-inlet azimuthal vorticity considered by Rusak et al. (1998). These studies are in progress.

This research was supported by ARC grant 89927007, and RFBR grants 96-0101949 and 97-01-00773, associated with Oleg Derzho affiliation with Inst. Thermophysics, Novosibirsk, Russian Academy of Science.

\section{Appendix}

The exact form of the coefficients in (3.12) for the velocity and swirl profiles given by (3.11) and $\Omega_{0}=\frac{1}{2} \lambda^{(0)}$ is the following:

$$
\begin{aligned}
\alpha_{1}= & 0.333\left[-\hat{\kappa}\left(8 a_{2}+2 \lambda^{(0)^{2}} a_{1}\right)+3 \lambda^{(0)^{2}} \hat{\sigma} b_{1}\right]-0.291 \lambda^{(0)^{2}}\left(\hat{\kappa} a_{2}-2 \hat{\sigma} b_{2}\right) \\
& +2 \lambda^{(0)^{2}} \hat{\kappa}\left(a_{1}+a_{2}\right)-2 \lambda^{(0)^{2}} \hat{\sigma}\left(b_{1}+b_{2}\right) \\
\alpha_{2}= & 0.394 \lambda^{(0)^{2}}\left(-\hat{\kappa} a_{1}+2 \hat{\sigma} b_{1}\right)+0.277 \lambda^{(0)^{2}}\left(-\hat{\kappa} a_{2}+\frac{8}{3} \hat{\sigma} b_{2}\right), \\
\alpha_{3}= & 0.295 \lambda^{(0)^{2}}\left(-\hat{\kappa} a_{2} / 3+\hat{\sigma} b_{2}\right) .
\end{aligned}
$$

\section{REFERENCES}

Aigner, A. 2001 Numerical simulations of internal and inertial solitary waves. $\mathrm{PhD}$ thesis, Monash University.

Aigner, A., Broutman, D. \& Grimshaw, R. 1999 Numerical simulations of internal solitary waves with vortex cores. Fluid Dyn. Res. 25, 315-333.

Akylas, T. R. \& Grimshaw, R. 1992 Solitary internal waves with oscillatory tails. J. Fluid Mech. 242, 279-298.

BATCHELOR, G. K. 1956 On steady laminar flow with closed streamlines at large Reynolds numbers. J. Fluid Mech. 1, 177-190.

Benjamin, T. B. 1962 Theory of the vortex breakdown phenomenon. J. Fluid Mech. 14, 593-629.

Benjamin, T. B. 1967 Some developments in the theory of vortex breakdown. J. Fluid Mech. 28, 65-84.

BERAN, P. S. 1994 The time asymptotic behaviour of vortex breakdown in tubes. Comput. Fluids 23, 917-937.

Beran, P. S. \& Culick, F. E. C. 1992 The role of non-uniqueness in the development of vortex breakdown in tubes. J. Fluid Mech. 242, 491-527.

BragG, S. L. \& Hawthorne, W. R. 1950 Some exact solutions of the flow through annular cascade actuator discs. J. Aero. Sci. 17, 243-249.

Brown, G. L. \& Lopez, J. M. 1990 Axisymmetric vortex breakdown. Part 2. Physical mechanisms. J. Fluid Mech. 221, 553-576.

Buntine, J. D. \& Saffman, P. G. 1995 Inviscid swirling flows and vortex breakdown. Proc. R. Soc. Lond. A 449, 139-153.

Clarke, S. R. \& Grimshaw, R. H. J. 1999 The effect of weak shear on finite amplitude internal solitary waves. J. Fluid Mech. 395, 125-159.

DARMOFAL, D. L. 1996 Comparisons of experimental and numerical results for axisymmetric vortex breakdown in pipes. Comput. Fluids 25, 353-371. 
Derzho, O. G. 1992 The propagation of solitary waves in a stratified shear flow with a free boundary. Proc. Intl Symp. Heat Mass Transfer, Bialowieza, 113-120.

Derzho, O. G. \& Grimshaw, R. 1997 Solitary waves with a vortex core in a shallow layer of stratified fluid. Phys. Fluids 9, 3378-3385.

Escudier, M. P. 1988 Vortex breakdown. Observations and explanations. Prog. Aerosp. Sci. 25, 189-229.

FALER, J. H. \& Leibovich, S. 1978 An experimental map of the internal structure of a vortex breakdown. J. Fluid Mech. 86, 313-333.

Fraenkel, L. E. 1964 The non-existence of a suitable solution for inviscid flow of type A. Appendix to J. B. Benjamin and B. J. S. Barnard. J. Fluid Mech. 19, 193.

Goldshtik, M. \& Hussain, F. 1997 The nature of inviscid vortex breakdown. Phys. Fluids 9, 263-265.

Grimshaw, R. \& YI, Z. 1993 Resonant generation of finite amplitude waves by the uniform flow of a uniformly rotating fluid past an obstacle. Mathematika 40, 30-50.

HANAZAKI, H. 1996 On the wave excitation and the formation of recirculation eddies on axisymmetric flow of uniformly rotating fluids. J. Fluid Mech. 322, 165-200.

Keller, J. J. 1995 On the interpretation of vortex breakdown. Phys. Fluids 7, 1695-1702.

Keller, J. J., Egli, W. \& Althaus, R. 1988 Vortex breakdown as a fundamental element of vortex dynamics. Z. angew. Math. Phys. 39, 404-439.

Keller, J. J., Egli, W. \& Exley, J. 1985 Force and loss-free transitions between flow states. Z. angew. Math. Phys. 36, 854-889.

Kopecky, R. M. \& TorRANCE, K. E. 1973 Initiation and structure of axisymmetric eddies in a rotating stream. Comput. Fluids 1, 289-300.

LeIBovich, S. 1970 Weakly nonlinear waves in rotating fluids. J. Fluid Mech. 42, 803-822.

LeIbovich, S. 1984 Vortex stability and breakdown: survey and extension. AIAA J. 22, 1192-1206.

LeIBovich, S. 1991 Vortex breakdown: a coherent transition trigger in concentrated vortices. In Turbulence and Coherent Structures (ed. O. Metais \& M. Lesieur), pp. 285-302. Kluwer.

Leibovich, S. \& Kribus, A. 1990 Large amplitude wavetrains and solitary waves in vortices. J. Fluid Mech. 216, 459-504.

Leibovich, S. \& Randall, J. D. 1973 Amplification and decay of long linear waves. J. Fluid Mech. 53, 481-493.

LONG, R. R. 1953 Steady motion around a symmetrical obstacle moving along the axis of a rotating liquid. J. Met. 10, 197-203.

Lopez, J. M. 1990 Axisymmetric vortex breakdown. Part 1. Confined swirling flow. J. Fluid Mech. 221, 533-552.

LOPEZ, J. M. 1994 On the bifurcation structure of axisymmetric vortex breakdown in a constricted pipe. Phys. Fluids 6, 3683-3693.

Pritchard, W. G. 1969 The motion generated by a body moving along the axis of a uniformly rotating fluid. J. Fluid Mech. 39, 443-464.

Rottman, J. W., Broutman, D. \& Grimshaw, R. H. J. 1996 Numerical simulations of uniformly stratified flow over topography. J. Fluid Mech. 306, 1-30.

RUSAK, Z. 1998 The interaction of near-critical swirling flows in a pipe with inlet azimuthal vorticity perturbations. Phys. Fluids 10, 1672-1684.

Rusak, Z., Wang, S. \& Whiting, C. H. 1998 The evolution of a perturbed vortex in a pipe to axisymmetric vortex breakdown. J. Fluid Mech. 366, 211-237.

SARPKAYA, T. 1971 On stationary and travelling vortex breakdown. J. Fluid Mech. 45, 545-559.

SNYDER, D. O. \& SPALl, R. E. 2000 Numerical simulation of bubble type vortex breakdown within a tube-and-vane apparatus. Phys. Fluids 12, 603-608.

Squire, H. B. 1956 Rotating fluids. In Surveys in Mechanics (ed. G. K. Batchelor \& R. M. Davies), pp. 139-161. Cambridge University Press.

WANG, S. \& RuSAK, Z. 1996 On the stability of non-columnar swirling flows. Phys. Fluids 8, $1017-1023$.

WANG, S. \& Rusak, Z. $1997 a$ The dynamics of a swirling flow in a pipe and transition to axisymmetric vortex breakdown. J. Fluid Mech. 340, 177-223.

WANG, S. \& RUSAK, Z. $1997 b$ The effect of slight viscosity on a near-critical swirling flow in a pipe. Phys. Fluids 9, 1914-1927. 